\title{
Analytical and numerical study of the stress field in a circular semi- ring under combined diametral compression and bending
}

\author{
Stavros K. Kourkoulis, Ermioni D. Pasiou, Christos F. Markides \\ National Technical University of Athens, School of Applied Mathematical and Physical Sciences, Department of Mechanics, \\ Laboratory for Testing and Materials, 5, Heroes of Polytechnion Avenue, Zografou Campus, Theocaris Building, 157 73, Greece \\ stakkour@central.ntua.gr,epasion@teemail.gr,markidib@mail.ntua.gr
}

\begin{abstract}
The stress field developed in a circular semi-ring under the combined action of diametral compression and bending is explored both analytically and numerically. The analytic solution is implemented by means of the complex potentials technique as it was formulated by Muskhelishvili, while for the numerical study a finite element model, properly validated based on experimental data, is used. The analytic solution provided closed formulae for the stress field along strategic loci of the specimen, while the numerical model permitted thorough parametric investigation of the dependence of critical quantities on geometrical and loading factors. The idea behind the study is to assess the potentialities of the circular semi-ring as a possible substitute of the familiar Brazilian disc, in the direction of curing drawbacks of the latter. It was concluded that a circular semi-ring subjected to eccentric diametral compression provides reliable data for the tensile strength of very brittle materials, relieved from ambiguities characterizing the standardized Brazilian-disc test.
\end{abstract}

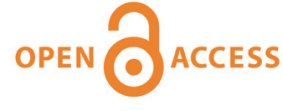

Citation: Kourkoulis S.K., Pasiou E.D, Markides Ch.F., Analytical and numerical study of the stress field in a circular semi-ring under combined diametral compression and bending, Frattura ed Integrità Strutturale, 47 (2019) $247-265$.

Received: 21.08 .2018

Accepted: 28.10 .2018

Published: 01.01.2019

Copyright: (C) 2019 This is an open access article under the terms of the CC-BY 4.0, which permits unrestricted use, distribution, and reproduction in any medium, provided the original author and source are credited.

KEYwORDs. Circular semi-ring; Brazilian-disc test; Tensile strength; Complex potentials; Finite element method; Stress field.

\section{INTRODUCTION}

$\mathrm{T}$ he implementation of standardized uniaxial tensile tests, with specimens made of brittle materials (as for example various hard rocks and rock-like geomaterials), encounters insuperable difficulties, related mainly to local fragmentation of the specimens during gripping and, also, fracture of the specimens in the immediate vicinity of the gripping area (rendering the data of the tests unreliable, given that the stress field in these regions is not purely uniaxial). In an attempt to overcome the problem, the engineering community is long ago seeking for alternative configurations that could provide at least an estimation of the uniaxial tensile strength of very brittle structural materials. Among the alternative configurations proposed, the one most widely accepted worldwide, is that of a circular disc compressed by diametral forces, 
which is known as Brazilian-disc test, honouring the Brazilian engineer Fernando Carneiro [1] who was the first one that proposed the specific configuration as a substitute of the direct tension test, already since 1943 (almost simultaneously with the Japanese engineer Tsunei Akazawa [2]).

In spite of its wide acceptance (mainly due to the simplicity of the geometry of the specimens and of the experimental setup), the Brazilian-disc test was strictly criticized almost immediately after it was introduced. The main points raising concerns about the validity of its outcome (and the relation of the quantity obtained with the tensile strength determined from a uniaxial tension test) are related to the fact that the stress field at the center of the disc is biaxial rather than uniaxial and, also, to the fact that (under specific conditions) fracture may originate from the immediate vicinity of the load application area (the vicinity of the points where the load is imposed) rather than from the center of the disc (rendering the validity of solutions [3-9] providing the "tensile strength" questionable [10-14]). In this direction, a variety of alternative configurations were gradually introduced, each one with its own pro and cons. Among them one should mention the ring test [15, 16], the flattened Brazilian-disc test [17] and the semi-circular bend test [18].

In the present study an alternative configuration is introduced, curing some drawbacks of previous attempts. The configuration proposed is that of a circular semi-ring (outer radius $R_{2}$, inner radius $R_{1}$, thickness 2 h), which is loaded under compression, as it is shown in Fig. 1a. The respective test will be denoted from here on as the Circular Semi-Ring test (CSR test). The main advantage of the specific test is that the stress field at the critical point A (i.e., the point at which fracture is expected to start) includes a single tensile component. In addition, the ratio between the maximum tensile stress (developed at point A) and the respective compressive one (developed at point B) is relatively easily controlled by the ratio $\varrho=R_{2} / R_{1}$. Moreover, the force required to cause fracture of the specimens is relatively low (compared to other more compact configurations). For the sake of generality, a non-zero eccentricity $c$ of the applied load $P$, with respect to the vertical $y$-axis, has been considered (obviously, one can always assume $c=0$, simplifying significantly the analytic calculations). The similarity of the CSR-configuration to the familiar arc-shaped notched tension specimen, proposed by ASTM [19] for the standardized determination of Mode-I fracture toughness (ASTM E399-90 standard), is to be highlighted.

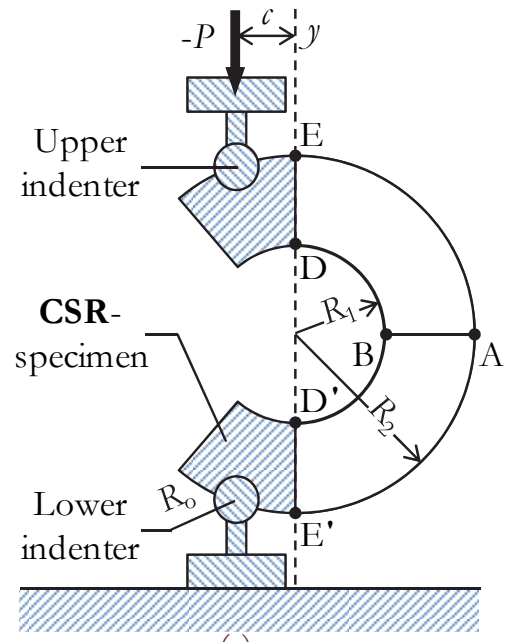

(a)

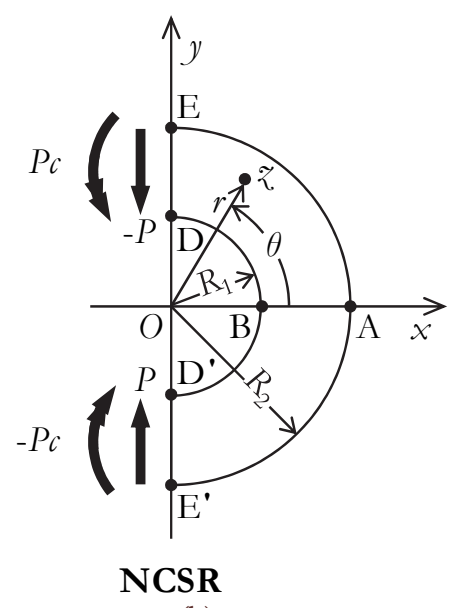

(b)

Figure 1: (a) The configuration proposed for the experimental implementation of the CSR-test; (b) The NCSR configuration considered in the analytic solution of the problem.

\section{ANALYTICAL CONSIDERATIONS}

he stress field in the CSR will be here obtained analytically by adopting Muskhelishvili's solution for a curved beam [20], assuming that the material of the CSR is homogeneous, isotropic and linearly elastic. It is emphasized from the very beginning that, the configuration considered in the analytic solution of the problem (shown in Fig. 1b) is somehow simplified compared to that of Fig. 1a, which is, in fact, proposed for the laboratory implementation of the test. The configuration of Fig. 1b will be denoted from here on as Net Circular Semi-Ring (NCSR), in order to be distinguished from that of Fig. 1a. In this context, and taking into account the eccentricity $c$ of the externally applied loading with respect to $y$-axis (which, for practical reasons, is very difficult to be avoided during the laboratory implementation of the experiments), the analytic solution deals with an NCSR, simultaneously subjected to bending by transverse forces $-P, P$ and couples $P c,-P c$ applied to its straight edges ED and E'D' (Fig. 1b). 
In spite of the above mentioned difference between the CSR-specimen and the NCSR-configuration, the theoretically predicted stresses in the vicinity of the critical cross-section $\mathrm{AB}$ (which is in fact the area of major interest for engineering applications) are expected to approach well the respective ones developed in the CSR-specimen, provided that the width $(w=$ $R_{2}-R_{1}$ ) of the NCSR is relatively small and that the resultant force and moment considered at its straight edges can efficiently replace the shaded parts of the CSR-specimen (Saint Venant's principle). As it will be seen by the comparative consideration of the results of the numerical and the analytical approaches, the above assumptions are well justified even in case the inner radius, $R_{1}$, of the CSR-specimen is equal to half of its outer one, $R_{2}$.

The analytic solution described in next sections is deduced from the respective one of the circular ring (CR) (in fact the NCSR is here considered as part of the CR [20]), under the admission of multi-valued displacements and the concept of dislocation, shortly recapitulated in the next paragraph. It is here recalled that the specific way of approaching this family of problems was first introduced by Golovin [21] (together with a series of solutions for the problem of curved beams under various loading schemes).

\section{Characteristics of the dislocation}

In this section a brief outline of the concepts of multi-valued displacements and dislocation will be given (together with a short description of the method adopted for obtaining the stress field in the NCSR through the solution of the respective CR problem), as they were analyzed in Muskhelishvili's milestone book [20]. In this context, a homogeneous, isotropic and linearly elastic $C R$, of inner and outer radii $R_{1}$ and $R_{2}$, respectively, is considered in equilibrium under an arbitrary in-plane loading scheme.

Assuming that the cross-section of the CR lies in the $z=x+\mathrm{i} y=r \mathrm{e}^{\mathrm{i} \theta}$ plane, with its centre at the origin of the Cartesian reference system, Muskhelishvili's general solution for the first fundamental problem for the CR is written as [20]:

$$
\Phi(z)=A \log z+\sum_{-\infty}^{+\infty} a_{k} z^{k}, \quad \Psi(z)=\sum_{-\infty}^{+\infty} a_{k}^{\prime} z^{k}
$$

The demand for the displacements to be single valued in the CR reads as [20]:

$$
u^{+}-u^{-}=0, \quad v^{+}-v^{-}=0
$$

where $u$ and $v$ are the horizontal and vertical components of the displacement field, respectively, with $(+)$, $(-)$ indicating the two sides of a cut joining the outer and inner perimeters of the CR, converting it into a simply connected region (Figs. 2 and 3). Constants $a_{k}, a_{k}{ }^{\prime}$ in Eqs.(1) (excluding the imaginary part of $a_{0}$ that remains arbitrary) are determined from the fulfillment of the boundary conditions for stresses and the following conditions, resulting from Eqs.(2) [20]:

$$
A=0, \quad \kappa a_{-1}+\vec{a}_{-1}^{\prime}=0 \quad\left(\kappa=\frac{\lambda+3 \mu}{\lambda+\mu}, \quad \lambda=\left\{\begin{array}{c}
E v /[(1+v)(1-2 v)], \text { plane strain } \\
E v /\left(1-v^{2}\right), \text { plane stress }
\end{array}\right)\right.
$$

In Eqs.(3), E is the Young's modulus, $v$ the Poisson's ratio and $\mu$ the shear modulus. Over-bar denotes the complex conjugate value. In case, now, multi-valued displacements are permitted in the cut CR, then instead of Eqns. (2) it holds that [20]:

$$
u^{+}-u^{-}=-\varepsilon y+\alpha, \quad v^{+}-v^{-}=\varepsilon x+\beta
$$

In this context, the additional expressions, besides the boundary conditions, for obtaining $a_{k}, a_{k}{ }^{\prime}$ of Eqns. (1) (except the imaginary part of $a_{0}$ ) are given (instead by Eqns. (3)) by the following expressions [20]:

$$
A=\frac{\pi(1+\kappa)}{\mu \varepsilon}, \quad \kappa a_{-1}+\vec{a}_{-1}^{\prime}=\frac{\mu}{\pi \mathrm{i}}(\alpha+\mathrm{i} \beta)
$$

In Eqs.(4) and (5), $\varepsilon$ (with dimensions of angle), and $a, \beta$ (with dimensions of length), are arbitrary, infinitesimal, real constants, expressing the relative angle of rigid body rotation of the two sides of the cut about the origin, and the relative rigid 
body translation of the two sides of the cut along $x$ - and $y$-axis, respectively (Figs. 2 and 3). The difference from the previous case (Eqs. $(2,3))$ is that by admitting the above multi-valued displacements in the cut CR, gaps or/and overlaps will appear

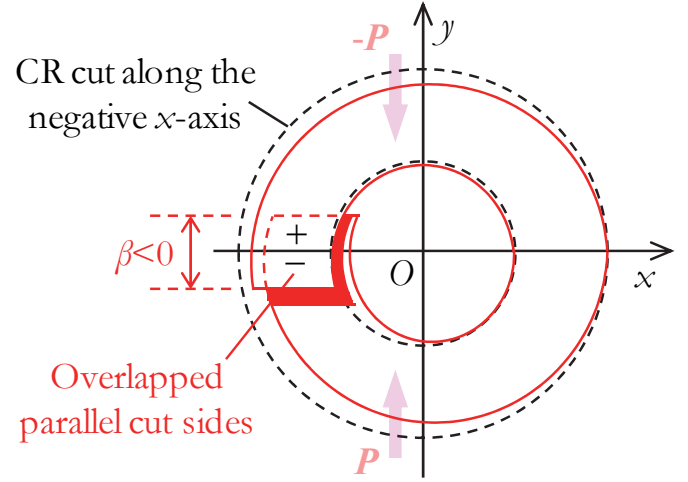

(a)

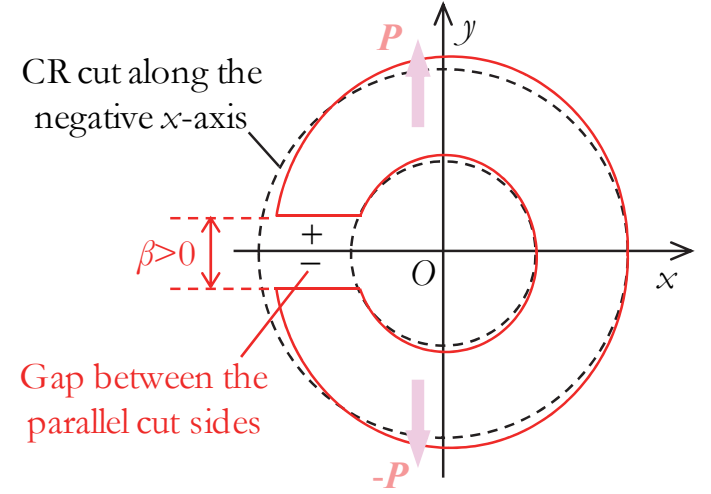

(b)

Figure 2: The nature of the $\beta$-dislocation and the resulting transverse forces for (a) negative and (b) positive values.

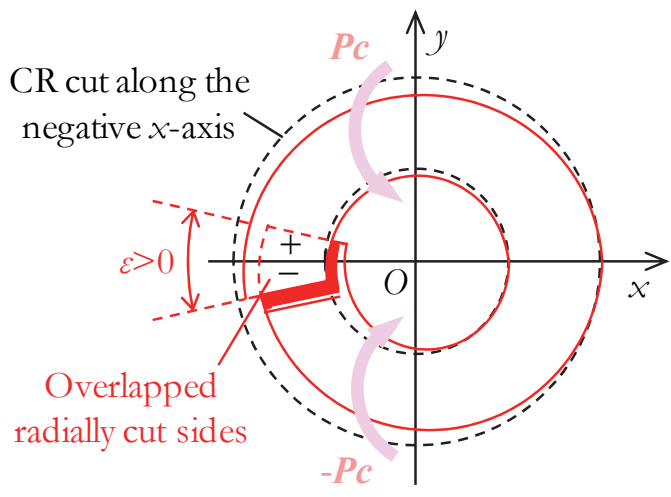

(a)

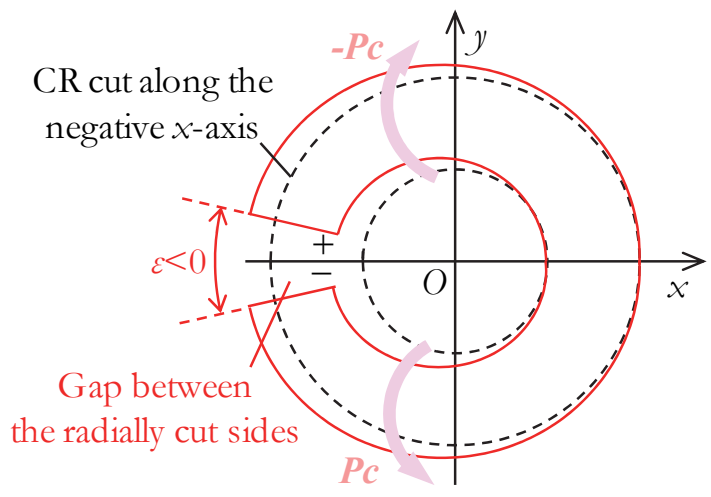

(b)

Figure 3: The nature of the $\varepsilon$-dislocation and the resulting couples for (a) positive and (b) negative values.

between the sides of the cut, dictated by the values of $\varepsilon, a$ and $\beta$ and stresses will be developed within the cut CR, even in case of zero external forces. These stresses, due to $\varepsilon, a$ and $\beta$ (and not due to the application of some external loading scheme), are of particular interest in this study and will actually provide the stress field in the NCSR-configuration and in turn in the CSR-specimen in question, at least approximately.

Before dealing with these stresses (as it is thoroughly described in next section), a few comments must be made for clarity on the nature of multi-valued displacements within the cut CR, which are due to Muskhelishvili [20]. Namely, it is mentioned that $\varepsilon, a$ and $\beta$ are the so-called "characteristics of the dislocation" (as named by Love [22]) and it was Timpe [23] who gave first a physical interpretation to these quantities in the case of the CR (later Volterra [24] dealt with the general case of multiply connected regions). Timpe [23] stated that even allowing multi-valued displacements, deformation can still be compatible assuming that one adds to (or/and removes from) the cut CR, strips of the same material, of dimensions $\varepsilon, a$ and $\beta$ and then rejoin the cut CR in one part. Obviously, when $\varepsilon, a$ and $\beta$ are to cause a gap between the sides of the cut (Figs. ( $2 \mathrm{~b}, 3 \mathrm{~b})$ ), a strip should be added between the sides of the cut and joined to them, whereas when $\varepsilon, a$ and $\beta$ are to cause overlapping of the sides of the cut (Figs. (2a, 3a)), a strip should be removed from the sides of the cut and the generated new free sides of the cut should be brought into contact and joined together. In this way, the resulting CR will remain continuous after any actual deformation due to a non-zero external loading scheme, without gaps or overlaps, since the two sides of the cut will move as a single unit.

\section{The stress field in the NCSR due to the characteristics of the dislocation}

The next step is to return back to the initial problem, in order to describe the way the "characteristics of the dislocation" $\varepsilon$, $a$ and $\beta$ can provide the stress field in the CSR-specimen. As it was mentioned in previous section, the stress field in the CSR-specimen may (under certain conditions meeting Saint Venant's Principle) be provided approximately from the solution of the problem of the NCSR subjected simultaneously to transverse forces $-P, P$ and couples $P c,-P c$ at its straight 
edges ED and E'D' (Fig. 1b). On the other hand, as it will be shown below, suitable combinations of $\varepsilon, a$ and $\beta$ lead to effective solutions to the problems of the NCSR under transverse forces and couples at its straight edges, separately.

In this context, two independent problems are considered next for the NCSR: (a) the problem of bending by transverse forces of magnitude $P$ applied to its straight edges ED, E'D' and (b) the problem of bending by couples of magnitude $P c$ applied to its straight edges ED, E' $D^{\prime}$. Then within the framework of linear elasticity, the solution of the whole problem for the NCSR, approximating that of the CSR, will be obtained by just superposing the solutions of these two problems.

\section{(a) Bending of the NCSR by transverse forces applied to its straight edges}

Assume that the cut CR is free from external forces and that $\varepsilon=a=0$ and $\beta \neq 0$. Then the deformed cut CR would attain one of the configurations of Fig. 2, depending on the sign of $\beta$, whereas stresses would occur in the cut CR due to $\beta$, given as [20]:

$$
\begin{aligned}
& \sigma_{r}^{(\beta)}=\frac{\beta \mu(\lambda+\mu)}{\pi(\lambda+2 \mu)}\left(\frac{1}{r}-\frac{r}{R_{1}^{2}+R_{2}^{2}}-\frac{R_{1}^{2} R_{2}^{2}}{R_{1}^{2}+R_{2}^{2}} \frac{1}{r^{3}}\right) \cos \theta \\
& \sigma_{\theta}^{(\beta)}=\frac{\beta \mu(\lambda+\mu)}{\pi(\lambda+2 \mu)}\left(\frac{1}{r}-\frac{3 r}{R_{1}^{2}+R_{2}^{2}}+\frac{R_{1}^{2} R_{2}^{2}}{R_{1}^{2}+R_{2}^{2}} \frac{1}{r^{3}}\right) \cos \theta \\
& \tau_{r \theta}^{(\beta)}=\frac{\beta \mu(\lambda+\mu)}{\pi(\lambda+2 \mu)}\left(\frac{1}{r}-\frac{r}{R_{1}^{2}+R_{2}^{2}}-\frac{R_{1}^{2} R_{2}^{2}}{R_{1}^{2}+R_{2}^{2}} \frac{1}{r^{3}}\right) \sin \theta
\end{aligned}
$$

Setting in Eqns. (6-8) $\theta= \pm \pi / 2$, it is seen that the only non-zero stress-component on the cross-sections ED and E' D'of the cut CR is $\tau_{r}(\beta)$. Moreover, integrating $\tau_{+} \theta^{(\beta)} 2 h \mathrm{~d} r$ over ED and $\mathrm{E}^{\prime} \mathrm{D}^{\prime}$, one can determine the transverse force acting on $\mathrm{ED}$ and $\mathrm{E}^{\prime} \mathrm{D}$ ' as follows [20]:

$$
P=2 b \int_{R_{1}}^{R_{2}} \tau_{r \theta}^{(\beta)}(\theta= \pm \pi / 2) \mathrm{d} r= \pm \beta \frac{2 h \mu(\lambda+\mu)}{\pi(\lambda+2 \mu)} \frac{\left(R_{1}^{2}+R_{2}^{2}\right) \log \frac{R_{2}}{R_{1}}-R_{2}^{2}+R_{1}^{2}}{R_{1}^{2}+R_{2}^{2}}
$$

Then keeping from the CR the part between $\theta= \pm \pi / 2$, one obtains the NCSR configuration under transverse forces of magnitude $P(P>0)$ at its straight edges ED and E'D'. Moreover, as the fractions on the right-hand side of Eqn.(9) are always positive, the sign of $\beta$ will dictate the direction of $P$, and it is easily seen that for $\beta<0$, i.e., for (using Eqn.(9)):

$$
\beta=-\frac{P}{2 h} \frac{\pi(\lambda+2 \mu)}{\mu(\lambda+\mu)} \frac{R_{1}^{2}+R_{2}^{2}}{\left(R_{1}^{2}+R_{2}^{2}\right) \log \frac{R_{2}}{R_{1}}+R_{1}^{2}-R_{2}^{2}}<0
$$

one arrives at the first problem in question for the NCSR under bending by transverse forces $-P, P$ applied to its straight edges.

\section{(b) Bending of the NCSR by couples applied to its straight edges}

Assume that the cut CR is free from external forces and that $\varepsilon \neq 0, a=\beta=0$. In that case, the deformed cut CR would attain one of the configurations of Fig. 3, depending on the sign of $\varepsilon$ and stresses would appear in the cut CR due to $\varepsilon$, given as [20]:

$$
\begin{aligned}
& \sigma_{r}^{(\varepsilon)}=\frac{\varepsilon \mu(\lambda+\mu)}{\pi(\lambda+2 \mu)}\left(\log r+\frac{1}{r^{2}} \frac{R_{1}^{2} R_{2}^{2}}{R_{2}^{2}-R_{1}^{2}} \log \frac{R_{2}}{R_{1}}-\frac{R_{2}^{2} \log R_{2}-R_{1}^{2} \log R_{1}}{R_{2}^{2}-R_{1}^{2}}\right) \\
& \sigma_{\theta}^{(\varepsilon)}=\frac{\varepsilon \mu(\lambda+\mu)}{\pi(\lambda+2 \mu)}\left(\log r-\frac{1}{r^{2}} \frac{R_{1}^{2} R_{2}^{2}}{R_{2}^{2}-R_{1}^{2}} \log \frac{R_{2}}{R_{1}}-\frac{R_{2}^{2} \log R_{2}-R_{1}^{2} \log R_{1}}{R_{2}^{2}-R_{1}^{2}}+1\right) \\
& \tau_{r \theta}^{(\varepsilon)}=0
\end{aligned}
$$


From Eqns. (11-13), it is immediately seen that by considering any isolated part of the cut CR, one has a curved beam, the only non-zero stress-component on the straight edges of which is $\sigma_{\theta}^{(\varepsilon)}$. The distribution of that $\sigma_{\theta}^{(\varepsilon)}$, attaining its extrema at the beam's peripheries, is statically equivalent to counterbalancing couples at the beam's straight edges of magnitude [20]:

$$
2 h \int_{R_{1}}^{R_{2}} \sigma_{\theta}^{(\varepsilon)} r \mathrm{~d} r=\varepsilon \frac{h \mu(\lambda+\mu)}{2 \pi(\lambda+2 \mu)} \frac{\left(R_{2}^{2}-R_{1}^{2}\right)^{2}-4 R_{1}^{2} R_{2}^{2}\left(\log \frac{R_{2}}{R_{1}}\right)^{2}}{R_{2}^{2}-R_{1}^{2}}
$$

Taking into account that according to the assumptions adopted this magnitude should be equal to $P c$, then equating Eqn. (14) to Pc and solving for $\varepsilon$, one obtains:

$$
\varepsilon=\frac{2 P c}{h} \frac{\pi(\lambda+2 \mu)}{\mu(\lambda+\mu)} \frac{R_{2}^{2}-R_{1}^{2}}{\left(R_{2}^{2}-R_{1}^{2}\right)^{2}-4 R_{1}^{2} R_{2}^{2}\left(\log \frac{R_{2}}{R_{1}}\right)^{2}}>0
$$

It is seen that for the above positive value of $\varepsilon$, and by considering the part of the cut CR between $\theta= \pm \pi / 2$, one obtains the second problem in question for the NCSR under bending by couples $-P c, P c$ applied to its straight edges ED and E'D'.

\section{(c) The overall problem of the NCSR for bending by transverse forces and couples applied to its straight edges}

The overall stress field in the NCSR under transverse forces $-P, P$ and couples $P c,-\mathrm{P} c$ at its straight edges ED and E'D', approaching that of the CSR-specimen, is given by superposing the respective stress components: $\sigma_{r}=\sigma_{r}^{(\beta)}+\sigma_{r}^{(\varepsilon)}($ Eqns. $(6,11))$, $\sigma_{\theta}=\sigma_{\theta}^{(\beta)}+\sigma_{\theta}^{(\varepsilon)}$ (Eqns. $\left.(7,12)\right)$ and $\tau_{r \theta}=\tau_{r \theta}(\beta)+\tau_{r \theta}(\varepsilon)$ (Eqns. (8, 13)), with $\beta$ and $\varepsilon$ given by Eqs. $(10$, 15), respectively. It is mentioned that these stresses depend on the magnitude $P$ of the transverse force, the eccentricity $c$, the ratio $\varrho=R_{2} / R_{1}$, the thickness $2 b$ of the CSR, the loading conditions adopted (namely plane stress or plain strain), and, also, on the material (appearing in the expressions for the dislocations $\beta, \varepsilon$ ). It must be, also, mentioned that assigning to the parameters of the analysis arithmetic data corresponding to very brittle materials, the values calculated for $\beta$ and $\varepsilon$ (depending on the values of $P$ and $c$ considered) are extremely small, satisfying the principal assumptions governing the present study, i.e., the assumption of linear elasticity.

\section{Effectiveness of the analytic solution}

The effectiveness of the analytic solution was assessed in terms of the data for the displacement field, as it was obtained from a recent experimental protocol [25]. CSR-specimens made of PMMA ( $E=3.2 \mathrm{GPa}, \nu=0.36)$ with $2 h=10 \mathrm{~mm}, R_{1}=25 \mathrm{~mm}$, $R_{2}=50 \mathrm{~mm}$, and $c=6.25 \mathrm{~mm}$ were tested according to the scheme shown in Fig. $1 \mathrm{a}$. A $10 \mathrm{kN}$ electromechanical frame was used to load the specimens and the displacement field was measured with the aid of the Digital Image Correlation (DIC) technique. The experimental set-up is shown in Fig. 4a. All tests were quasi-static, implemented under displacement-control conditions, at a constant rate of $0.1 \mathrm{~mm} / \mathrm{min}$. A typical fractured specimen is shown in Fig. $4 \mathrm{~b}$. During loading, series of successive photos of the specimens were taken permitting the determination of the displacement field by means of the software of the DIC system. The experimental data for the displacements were then compared with the ones provided by the analytic solution (as it is analytically described by Markides et al. [25]), which read as:

(a) Displacements due to bending of the NCSR by transverse forces applied to its straight edges

$$
\begin{aligned}
u^{(\beta)}= & \frac{\beta(\lambda+\mu)}{4 \pi(\lambda+2 \mu)}\left\{\frac{\lambda+3 \mu-1}{\lambda+\mu} \log r-\left[\frac{\lambda+3 \mu}{\lambda+\mu} \frac{r^{2}}{R_{1}^{2}+R_{2}^{2}}+1-\right.\right. \\
& \left.\left.\frac{1}{\lambda+\mu} \frac{R_{1}^{2} R_{2}^{2}}{2\left(R_{1}^{2}+R_{2}^{2}\right)} \frac{1}{r^{2}}\right] \cos 2 \theta+\frac{2 r^{2}}{R_{1}^{2}+R_{2}^{2}}\right\}
\end{aligned}
$$




$$
v^{(\beta)}=\frac{\beta(\lambda+\mu)}{4 \pi(\lambda+2 \mu)}\left\{\frac{\lambda+3 \mu+1}{\lambda+\mu} \theta-\left[\frac{\lambda+3 \mu}{\lambda+\mu} \frac{r^{2}}{R_{1}^{2}+R_{2}^{2}}+1-\frac{1}{\lambda+\mu} \frac{R_{1}^{2} R_{2}^{2}}{2\left(R_{1}^{2}+R_{2}^{2}\right)} \frac{1}{r^{2}}\right] \sin 2 \theta\right\}
$$

(b) Displacements due to bending of the NCSR by couples applied to its straight edges

$$
\begin{aligned}
u^{(\varepsilon)}= & \frac{\varepsilon(\lambda+\mu)}{4 \pi(\lambda+2 \mu)}\left[\left(\frac{\lambda+3 \mu}{\lambda+\mu}-1\right)\left(\frac{1}{2}-\frac{R_{2}^{2} \log R_{2}-R_{1}^{2} \log R_{1}}{R_{2}^{2}-R_{1}^{2}}+\log r\right) r \cos \theta-\right. \\
& \left.\left(\frac{\lambda+3 \mu}{\lambda+\mu}+1\right) r \theta \sin \theta-\frac{\lambda+3 \mu}{\lambda+\mu} r \cos \theta-\frac{2 R_{1}^{2} R_{2}^{2}}{R_{2}^{2}-R_{1}^{2}} \log \frac{R_{2}}{R_{1}} \frac{\cos \theta}{r}\right] \\
v^{(\varepsilon)}= & \frac{\varepsilon(\lambda+\mu)}{4 \pi(\lambda+2 \mu)}\left[\left(\frac{\lambda+3 \mu}{\lambda+\mu}-1\right)\left(\frac{1}{2}-\frac{R_{2}^{2} \log R_{2}-R_{1}^{2} \log R_{1}}{R_{2}^{2}-R_{1}^{2}}+\log r\right) r \sin \theta+\right. \\
& \left.\left(\frac{\lambda+3 \mu}{\lambda+\mu}+1\right) r \theta \cos \theta-\frac{\lambda+3 \mu}{\lambda+\mu} r \sin \theta-\frac{2 R_{1}^{2} R_{2}^{2}}{R_{2}^{2}-R_{1}^{2}} \log \frac{R_{2}}{R_{1}} \frac{\sin \theta}{r}\right]
\end{aligned}
$$

The results of the comparison are plotted in Fig. $4 \mathrm{c}$, in which the displacement $u_{x} \equiv u$ developed along the AB locus (see Fig. 1a), is plotted according to both the analytic and the experimental data. The agreement is satisfactory almost all along the locus considered (which is, in fact the most crucial for practical applications), excluding a weak maximum recorded by the DIC-system in the immediate vicinity of point $\mathrm{B}$, which is not actually predicted by the analytic solution.

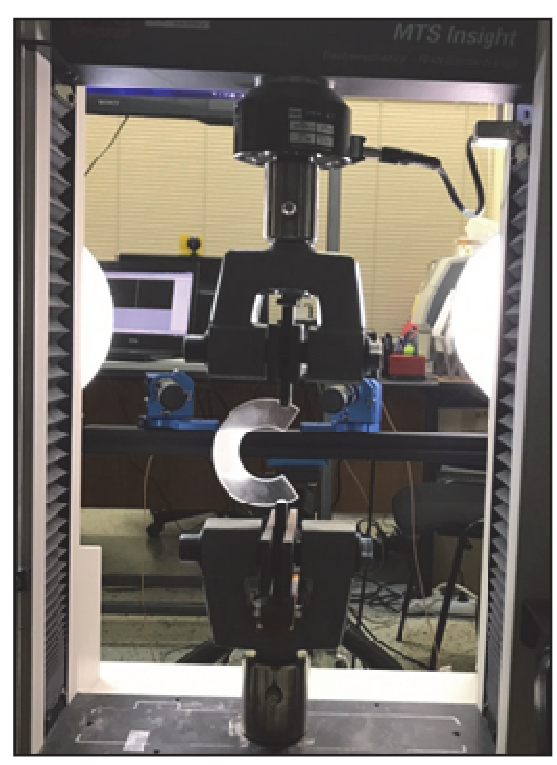

(a)

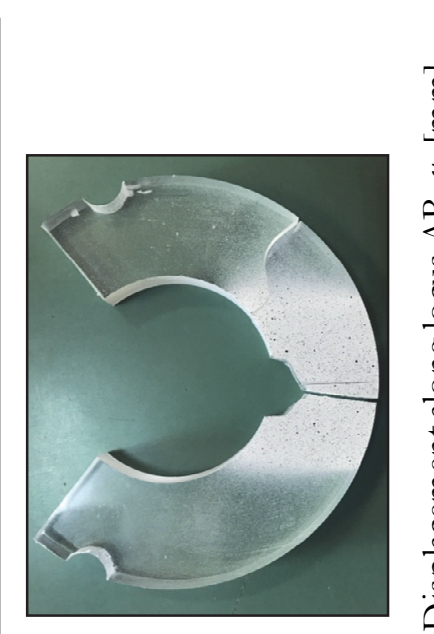

(b)

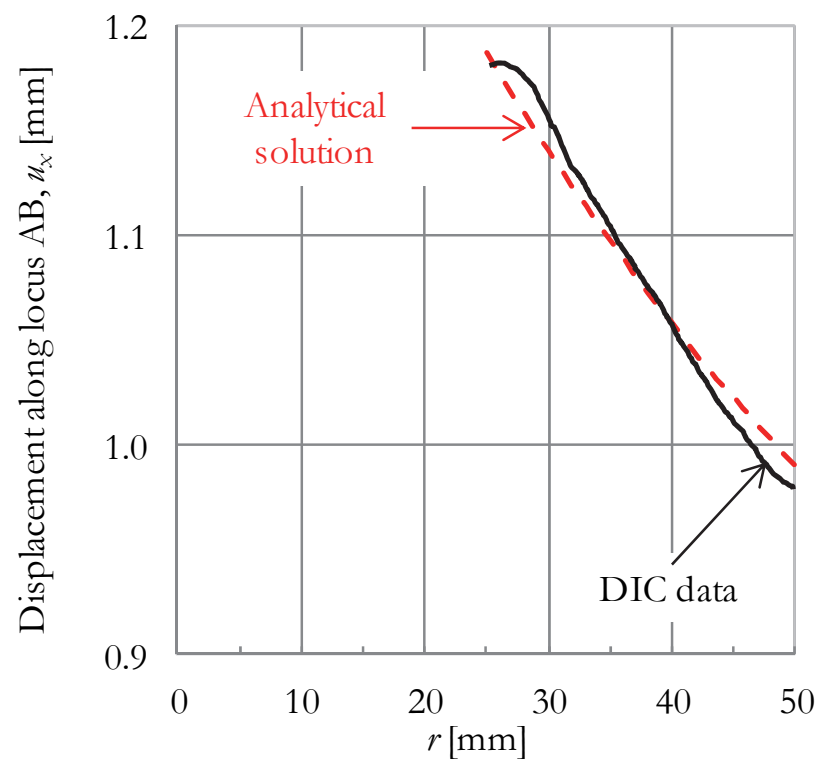

(c)

Figure 4: (a) An overview of the experimental set-up; (b) A fractured CSR specimen; (c) Analytic versus experimental data for the displacement component developed along the critical locus AB.

\section{The stress field in the NCSR at some strategic points and along some critical loci of the CSR}

In this paragraph the formulae obtained for the stress field in the NCSR are applied along some loci of particular practical (and engineering) interest. However, for a direct comparison between the data for the CSR-and the NCSR-configurations to be feasible, some preliminary remarks should be made. Initially, one should consider the conditions related to the eccentric application of the compressive forces (Fig. 1a) through the two cylindrical rods. Let $R_{0}$ be the radius of the rods and assume that half of their profile is inserted into the respective congruent hollow cavity of the CSR-specimen, at a given distance $c$ on the left of $y$-axis (Fig. 5). Then, the points of contact of the CSR with the indenters, corresponding to any specific eccentricity $c$, will be described as: 


$$
z=R_{c} e^{ \pm \mathrm{i}\left(\pi / 2+\Theta_{c}\right)}, R_{c}=\sqrt{R_{2}^{2}+R_{o}^{2}-2 R_{2} R_{o} \cos \theta_{o}}, \Theta_{c}=\theta_{o}+\tan ^{-1} \frac{R_{o} \sin \theta_{o}}{R_{2}-R_{o} \cos \theta_{o}}, \quad\left(\theta_{o}=\sin ^{-1} \frac{c}{R_{2}}\right)
$$

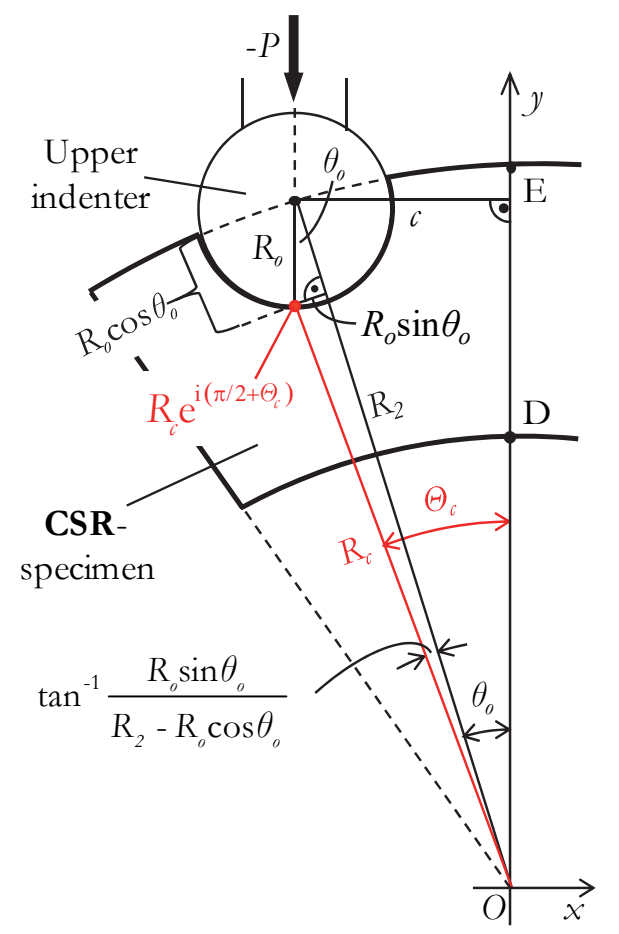

Figure 5: The procedure adopted to specify the contact point $z=R_{c} e^{i\left(\pi / 2+\Theta_{c}\right)}$.

Moreover, it is noted that during the experimental implementation of the CSR-test (as well as in the numerical model that will be described in next sections, which reproduces accurately the experimental procedure) both rods are restricted concerning their motion in the horizontal direction. In addition, the lower rod is fixed also concerning its vertical motion (i.e., it is assumed completely fixed, without any degree of freedom) and a vertical displacement $v_{P}$ is imposed to the CSR-specimen, with the aid of the upper rod. On the contrary, in the analytic study, diametral compressive forces $P$ and couples $P c$ are imposed on both vertical edges of the NCSR. Obviously, for comparison reasons, the magnitude of these forces and couples must produce a vertical displacement equal to $(1 / 2) v_{P}$ to the points where they are applied. In this direction, advantage is taken of the above mentioned formulae for the displacement field in the NCSR. More specifically, the NCSR is ideally extended to the CSR-configuration with the supporting parts shown shaded in Fig. 1a. The contact points $z=R_{c} \mathrm{e}^{ \pm \mathrm{i}\left(\pi / 2+\Theta_{c}\right)}$, mentioned previously, are assumed lying on the shadowed parts (which is always true since NCSR is part of the whole cut $\mathrm{CR}$, to which the analytic solutions obtained before refer to). Then, in accordance with the experimental procedure (and the respective numerical models), point $z=R_{c} \mathrm{e}^{-\mathrm{i}\left(\pi / 2+\Theta_{c}\right)}$ must be completely fixed while point $z=R_{c} \mathrm{e}^{+\mathrm{i}\left(\pi / 2+\Theta_{c}\right)}$ must be fixed only in the $x$-direction. For this to be achieved one should subtract from the analytically determined displacement field the respective displacements of the points $z=R_{c} \mathrm{e}^{ \pm \mathrm{i}\left(\pi / 2+\Theta_{c}\right)}$, considering them as rigid-body ones.

As a next step, the experimental/numerical value of $v_{P}$ must be introduced in the expression for the vertical displacement $v$ of point $z=R_{c} \mathrm{e}^{+\mathrm{i}\left(\pi / 2+\Theta_{c}\right)}$ providing the magnitude of the force $P$, corresponding to the specific value of $v_{P}$. Then, inserting this value of $P$ (as well as the proper value of $c$, i.e., the value matching the experimental/numerical configuration), into Eqs.(10,15), $\varepsilon$ and $\beta$ and in turn the theoretical stresses (Eqs.(6-8) and Eqs.(11-13)) in the NCSR are obtained, in a manner comparable to the experimental and numerical ones for the CSR-specimen.

In this context, and in order for some features of the analytical solution to be quantitatively enlightened, an NCSR made of PMMA ( $E=3.2 \mathrm{GPa}, v=0.36$ ) is here considered with $c=10 \mathrm{~mm}$ and $v_{P}=3 \mathrm{~mm}$ (corresponding to the data of the experimental protocol discussed previously and to the numerical models that will be described next). The force corresponding to these data (i.e., the force that has to be introduced in the analytic formulae for the stress field) is determined equal to $P=1188.3 \mathrm{~N}$ for plane strain conditions and to $P=994.5 \mathrm{~N}$ for plane stress ones. 
Adopting the above values the stress components at the two critical points of the configuration (namely points $A\left(R_{2}, 0\right)$ and $\left.B\left(R_{1}, 0\right)\right)$ are calculated equal to:

- $\sigma_{\theta}\left(R_{2}, 0\right)=39.97 \mathrm{MPa}, \sigma_{\theta}\left(R_{1}, 0\right)=-75.98 \mathrm{MPa}$, for plane strain,

- $\sigma_{\theta}\left(\mathrm{R}_{2}, 0\right)=33.45 \mathrm{MPa}, \sigma_{\theta}\left(\mathrm{R}_{1}, 0\right)=-63.59 \mathrm{MPa}$, for plane stress.

The ratio of the two stresses is equal to:

$$
\frac{\left|\sigma_{\theta}\left(R_{1}, 0\right)\right|}{\sigma_{\theta}\left(R_{2}, 0\right)}=1.9, \text { for } \rho=\frac{R_{2}}{R_{1}}=2
$$

In other words, the ratio of the maximum compressive stress developed over the respective maximum tensile one, in a CSR with $\varrho=2$, is less than 2. Compared to the respective ratio of the Brazilian disc test, which at the center of the disc is equal to 3, one of the major advantages of CSR-specimen is highlighted, since the CSR-test can be used in a broader variety of materials concerning the ratio of compressive over tensile strength.

Regarding now the contribution of each one of the two constituent components (transverse forces and bending moments) of the overall stress field, it can be determined that for plane strain conditions it holds that:

- Contribution of transverse forces, $-P, P$ ( $\beta$-dislocation):

- Contribution of couples, $-P c, P c$ ( $\varepsilon$-dislocation): while for plane stress it holds that:

- Contribution of transverse forces, $-P, P$ ( $\beta$-dislocation):

- $\quad$ Contribution of couples, $-P c, P c$ ( $\varepsilon$-dislocation):

In both cases (plane strain - plane stress), it is determined that:

- Point A:

- Point B:

In addition, for either plane strain or plane stress, it holds that:

- $\quad$ Bending by transverse forces, $-P, P$ ( $\beta$-dislocation):

$$
\begin{array}{ll}
\sigma_{\theta}^{(\beta)}\left(R_{2}, 0\right)=30.62 \mathrm{MPa}, & \sigma_{\theta}^{(\beta)}\left(R_{1}, 0\right)=-61.24 \mathrm{MPa}, \\
\sigma_{\theta}^{(\varepsilon)}\left(R_{2}, 0\right)=9.35 \mathrm{MPa}, & \sigma_{\theta}^{(\varepsilon)}\left(R_{1}, 0\right)=-14.74 \mathrm{MPa}, \\
\sigma_{\theta}^{(\beta)}\left(R_{2}, 0\right)=25.62 \mathrm{MPa}, & \sigma_{\theta}^{(\beta)}\left(R_{1}, 0\right)=-51.25 \mathrm{MPa}, \\
\sigma_{\theta}^{(\varepsilon)}\left(R_{2}, 0\right)=7.82 \mathrm{MPa}, & \sigma_{\theta}^{(\varepsilon)}\left(R_{1}, 0\right)=-12.34 \mathrm{MPa} .
\end{array}
$$

$$
\begin{array}{ll}
\sigma_{\theta}^{(\beta)}\left(R_{2}, 0\right)=0.77 \sigma_{\theta}\left(R_{2}, 0\right), & \sigma_{\theta}^{(\varepsilon)}\left(R_{2}, 0\right)=0.23 \sigma_{\theta}\left(R_{2}, 0\right), \\
\sigma_{\theta}^{(\beta)}\left(R_{2}, 0\right)=0.81 \sigma_{\theta}\left(R_{2}, 0\right), & \sigma_{\theta}^{(\varepsilon)}\left(R_{2}, 0\right)=0.19 \sigma_{\theta}\left(R_{2}, 0\right) .
\end{array}
$$

$$
\begin{aligned}
& \frac{\left|\sigma_{\theta}^{(\beta)}\left(R_{1}, 0\right)\right|}{\sigma_{\theta}^{(\beta)}\left(R_{2}, 0\right)}=\rho=\frac{R_{2}}{R_{1}}=2, \\
& \frac{\left|\sigma_{\theta}^{(\varepsilon)}\left(R_{1}, 0\right)\right|}{\sigma_{\theta}^{(\varepsilon)}\left(R_{2}, 0\right)}=\frac{\left|R_{2}^{2}-R_{1}^{2}-2 R_{2}^{2} \log \frac{R_{2}}{R_{1}}\right|}{R_{2}^{2}-R_{1}^{2}-2 R_{1}^{2} \log \frac{R_{2}}{R_{1}}}=1.58 .
\end{aligned}
$$

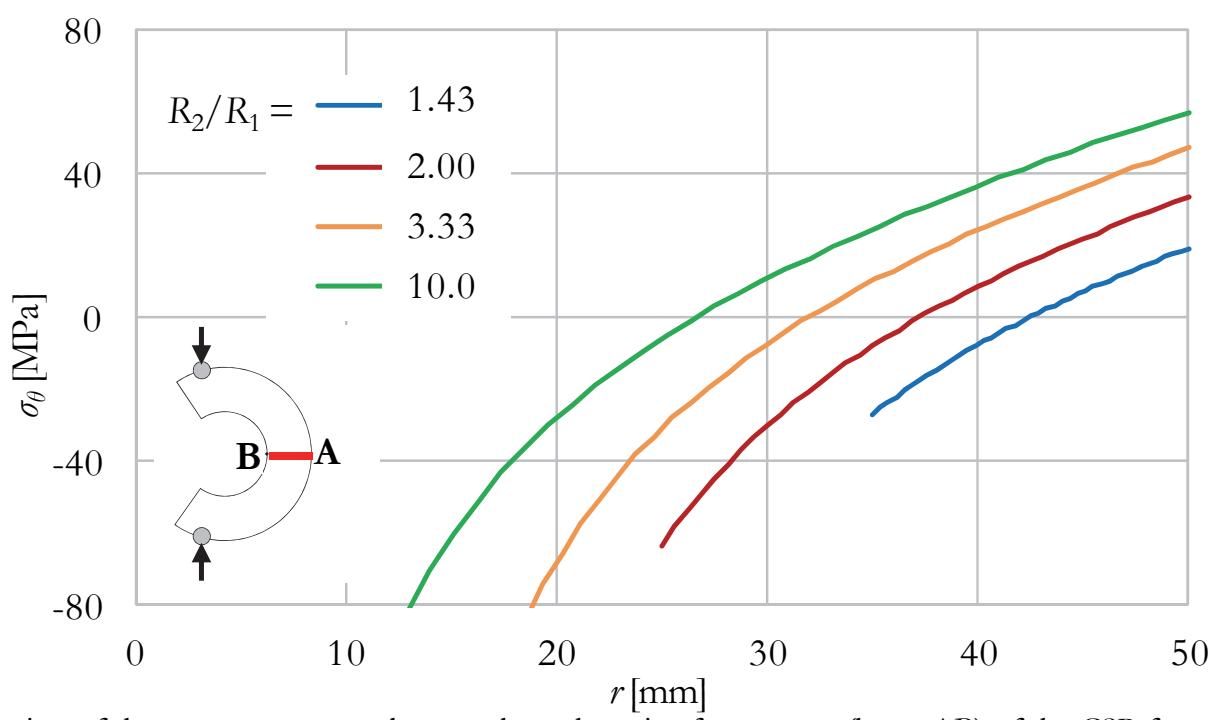

Figure 6: The distribution of the transverse normal stress along the axis of symmetry (locus AB) of the CSR for various $\varrho$-values. 
As it has been already mentioned, the ratio of the maximum compressive stress developed in a CSR over the respective maximum tensile one is controllable by a geometric parameter, namely the ratio $\varrho=R_{2} / R_{1}$ of the specimen's radii. In order to quantitatively explore this issue, the variation of the transverse stress, $\sigma_{\theta}$, along the locus $\mathrm{AB}$, is plotted in Fig. 6, for a series of values of the ratio $\varrho$, within the $1.43<\varrho<10.00$ range. It is seen from Fig. 6 that, the transverse stress is tensile (as it is expected) at the outermost point $\mathrm{A}$ of the locus $\mathrm{AB}$ and its value gradually decreases as one moves towards the innermost point $\mathrm{B}$. At a given $r$-value $\left(\mathrm{R}_{1}<r<\mathrm{R}_{2}\right)$ (which is not constant but rather it depends on $\varrho$ ), the stress is zeroed and then it becomes compressive of continuously increasing magnitude. As the CSR becomes thinner (i.e., for $R_{1}$ approaching $R_{2}$ ) the ratio of the maximum stresses at points $\mathrm{A}$ and $\mathrm{B}$ tends to 1 . Obviously, testing specimens with $\varrho$-values approaching unity is difficult; however, the fact that the maximum compressive stress developed is somehow controllable offers a serious advantage to the CSR-test against the standardized Brazilian-disc test for which such a control is not possible.

\section{NUMERICAL MODELING}

A $\mathrm{s}$ a next step, and in order to parametrically explore various aspects of the stress field, developed in a CSR-specimen under eccentric diametral compression, the finite element method is employed. In this direction, the commercially available software ANSYS (v.11) was used. The problem is studied in three dimensions and every effort was paid to properly simulate the configuration of Fig. 1a, i.e., the one proposed for the laboratory implementation of the CSR-test. A CSR with $R_{1}=25 \mathrm{~mm}, R_{2}=50 \mathrm{~mm}$ and thickness $2 b=10 \mathrm{~mm}$ (Figs. 1a and 7a) was considered as the reference model of the numerical study. In this context, two cylindrical rods were also constructed and placed in circular grooves (of the same diameter with that of the rods) at a distance equal to $c=10 \mathrm{~mm}$ from $y$-axis. All nodes of the lower cylinder were rigidly fixed and a vertical downwards displacement $v_{p}=3 \mathrm{~mm}$ was imposed on the nodes of the upper cylinder, the motion of which normally to $y$-axis is restricted. The specific value of the displacement imposed ensures that the material remains within its elastic regime during loading, as it has been indicated by a preliminary experimental protocol [25].

The mechanical properties assigned to the material of the CSR-specimen are those of PMMA (following the respective assignment of the analytical study, i.e., $E=3.20 \mathrm{GPa}, \nu=0.36$ ). The two load transferring cylinders were assumed to be made of steel $\left(E_{r}=210 \mathrm{GPa}, v_{r}=0.3\right)$. PMMA was modeled as linearly elastic material, taking into account that the CSR-test is proposed for the determination of the tensile strength of brittle geomaterials, the mechanical response of which is properly described within the frame of linear elasticity. Moreover, taking into account that the load required to cause fracture of a CSR-specimen is very small, compared to that causing yield of the rods, steel was also modeled as a linearly elastic material.

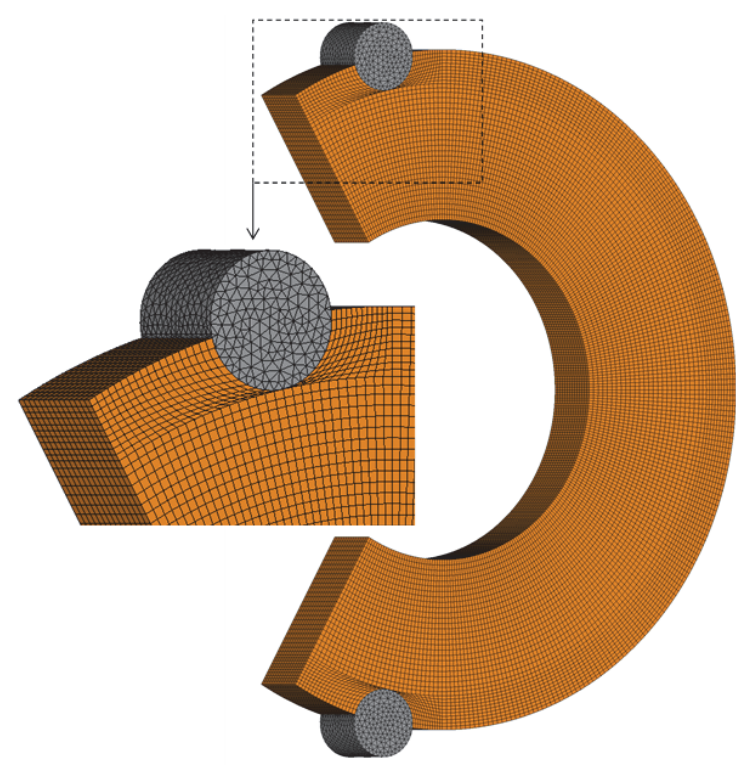

(a)

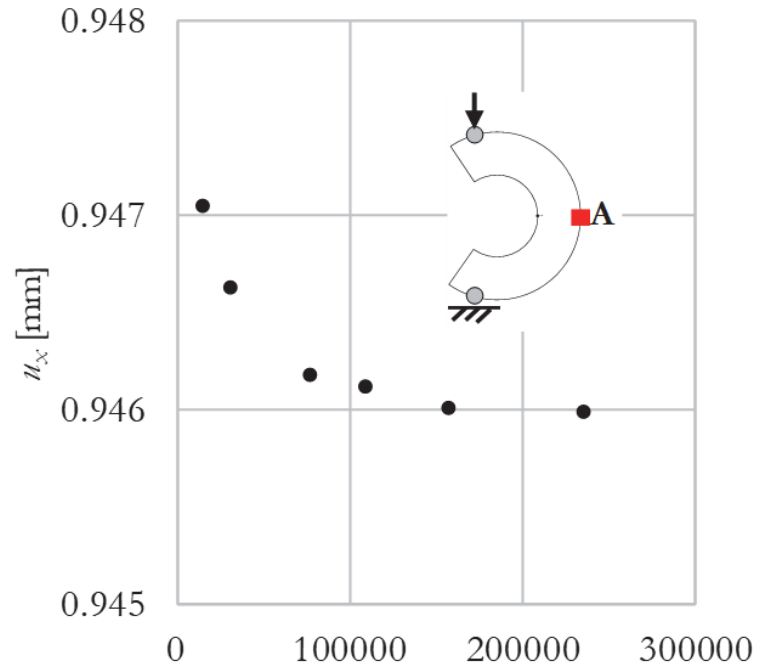

Number of elements

(b)

Figure 7: (a) The three dimensional numerical model properly meshed with 155000 elements; (b) The dependence of the horizontal displacement $u_{x} \equiv u$ of point $\mathrm{A}$ on the number of elements used for meshing the numerical model. 
Convergence and validation of the numerical model

SOLID185 element was used for meshing the model. The optimum element size was chosen after a proper convergence analysis. Initially, the horizontal displacement of point A was taken into account. Its dependence on the number of elements of the mesh is plotted in Fig. 7b. It is seen that using models with about 150000 elements offers a satisfactory compromise between accuracy and CPU-time consumption. In fact, the reference model finally used (shown in Fig. 7a) consists of about 155000 elements. The quality of convergence was further checked by considering the dependence of critical quantities along critical paths instead of single points. As such a path the locus $\mathrm{AB}$ was chosen. The variation of the maximum principal stress $\sigma_{1}$ along $\mathrm{AB}$ is plotted in Fig. 8 for models with increasing number of elements. It is seen that the differences between the models are worth noticing only in the immediate vicinity of point B and, also, at the point where the value of $\sigma_{1}$ changes sign. Again it is verified that models with about 150000 elements offer quite satisfactory accuracy.

For the validation of the numerical model to be achieved, the results of the reference model with 155000 elements, concerning the displacement field, are compared against the respective ones recorded during a recently implemented experimental protocol [25]. Attention is again focused to the critical path AB. The results for the horizontal displacement component are plotted in Fig. 9. It can be seen that, from a qualitative point of view, the agreement is excellent; the two curves (numerical and experimental) match almost perfectly to each other. From a quantitative point the difference between numerical and experimental data is less than $20 \mu \mathrm{m}$. In other words, the experimental data exceed the respective numerical ones by less than $2 \%$, all along the locus considered. Taking into account the very small magnitude of the experimentally measured quantities, the agreement between numerical and experimental data can be safely considered as excellent.

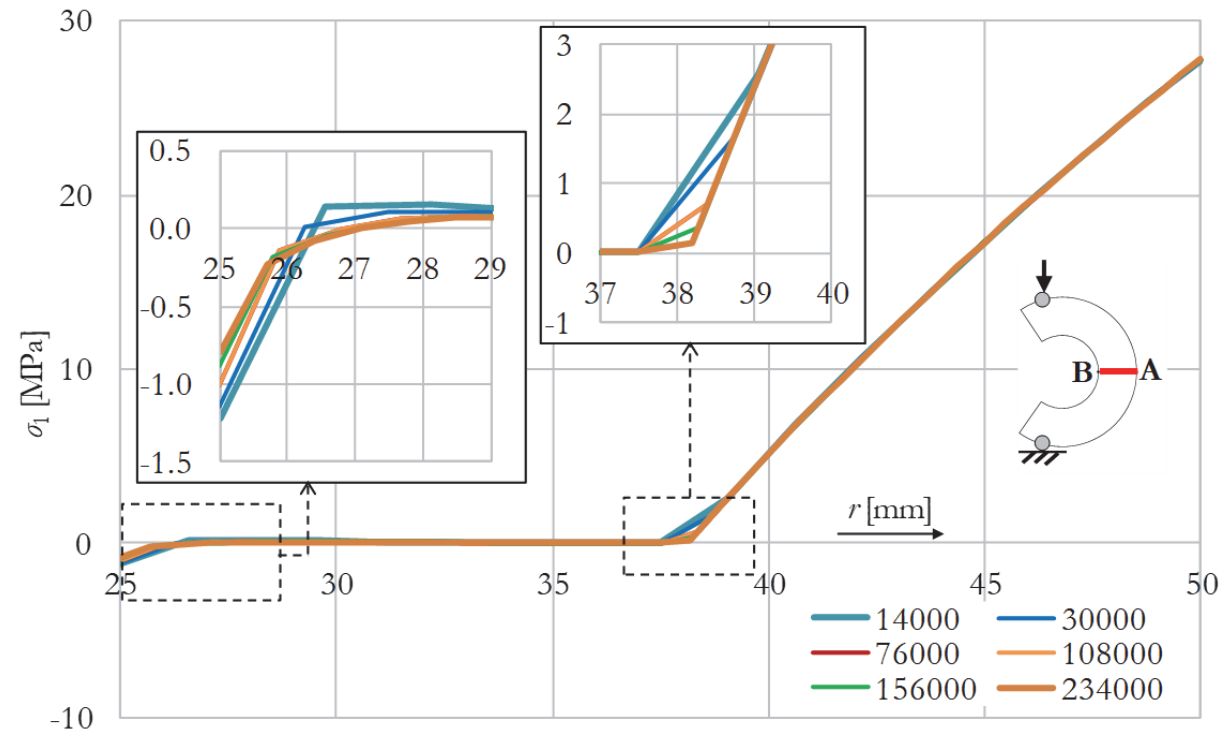

Figure 8: The dependence of the maximum principal stress $\sigma_{1}$, developed along the $\mathrm{AB}$ locus, on the number of elements used for meshing the reference model.

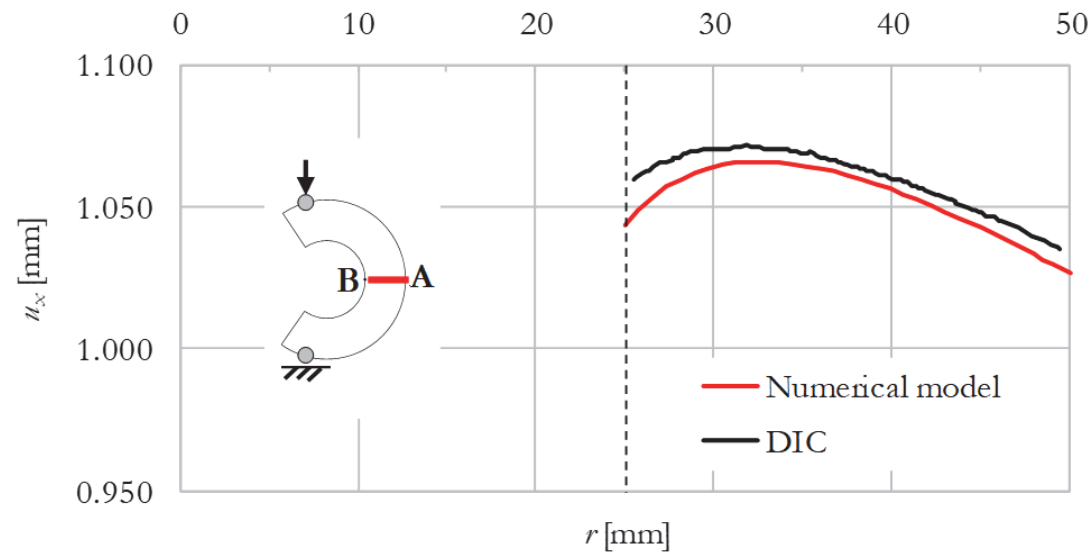

Figure 9: The horizontal component of the displacement field $u_{x} \equiv u$ developed along the AB locus determined numerically in juxtaposition to the respective one experimentally measured using the DIC technique [25]. 


$$
\frac{25}{21}
$$


The as above validated numerical model is now used to explore the role of some critical parameters on the stress field developed in CSR-specimens. Two parameters are considered in this first attempt, namely, the inner radius of the ring, $R_{1}$, and the horizontal distance of the load transferring rods from $y$-axis (in other words the eccentricity of the load applied). The values assigned to these parameters are recapitulated in Table 1 (bold numbers correspond to the reference model). The outer radius and the thickness of the ring are assumed constant equal to $R_{2}=50 \mathrm{~mm}$ and $2 h=10 \mathrm{~mm}$, respectively. From here on, for comparison reasons, a common vertical downwards displacement is imposed on the nodes of the upper load transferring rod, equal to $v_{p}=3 \mathrm{~mm}$, in all cases.

\begin{tabular}{ccccc}
\hline Parameter & \multicolumn{5}{c}{ Values } \\
$R_{1}[\mathrm{~mm}]$ & 5 & 15 & $\mathbf{2 5}$ & 35 \\
$c[\mathrm{~mm}]$ & 7.5 & $\mathbf{1 0 . 0}$ & 12.5 & 15.0 \\
\hline
\end{tabular}

Table 1: The numerical values assigned to the parameters studied in the numerical analysis.

The crucial role of the inner radius of the CSR on the respective stress field is clearly highlighted in Fig. 12, in terms of the $\varrho=R_{2} / R_{1}$ ratio. In Fig. 12a the tensile stress, $\sigma_{\theta, \mathrm{A}}$, developed at point A (namely the stress value, which at the fracture of the specimens is assumed to correspond to the tensile strength of the specimen's material) is plotted versus $\varrho$. It is observed that, for $\varrho$-values lower than $\varrho=3.0$ the specific stress component increases steadily with increasing $\varrho$. On the contrary, for $\varrho$ values exceeding $\varrho=3.0$, the value of $\sigma_{\theta, \mathrm{A}}$ tends to be stabilized at a value equal to about $\sigma_{\theta, \mathrm{A}} \approx 36 \mathrm{MPa}$. The dependence of the magnitude of the ratio of the maximum tensile stress over the respective compressive one, i.e., of the ratio of the stresses developed at points A and B, is plotted in Fig. 12b, again versus the $\varrho$-ratio. It is observed that the relation of the ratio of maximum stresses and the ratio of the radii is excellently described by a power law, almost all over the range of $\varrho$-values considered here.

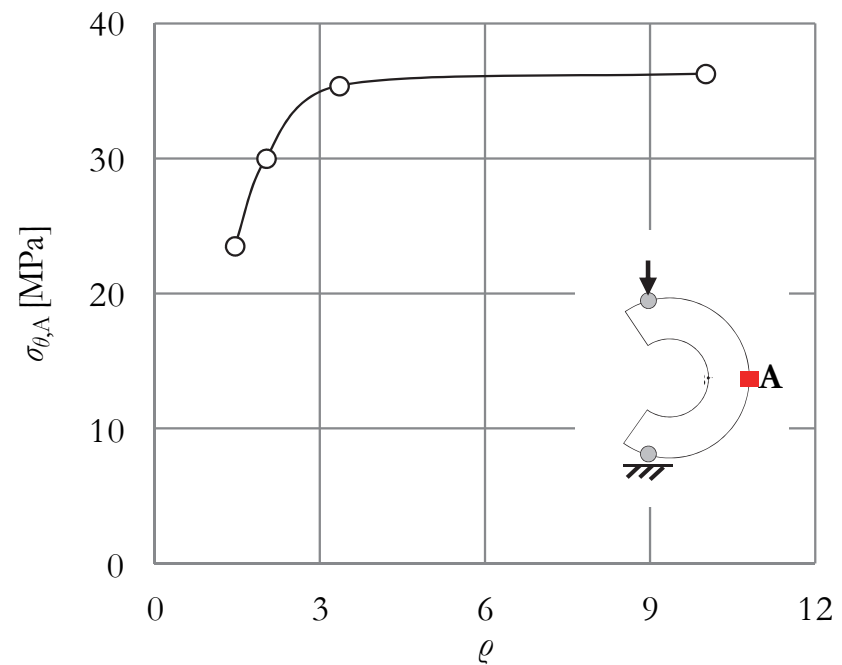

(a)

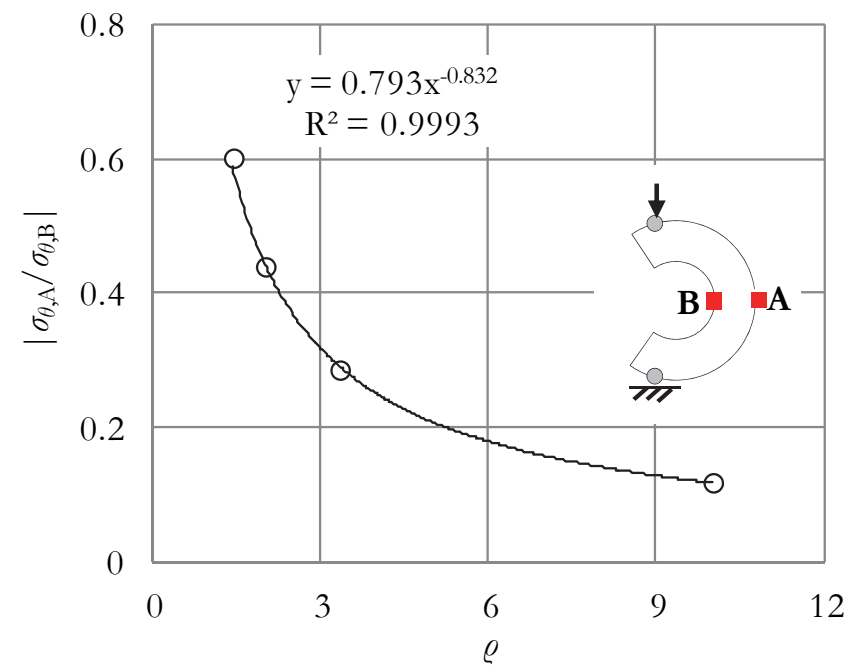

(b)

Figure 12: The dependence of the (a) maximum tensile stress and (b) the ratio of the maximum tensile stress over the respective compressive one, on the values of the radii ratio $\varrho$.

In Fig. 13 the polar distribution of the transverse stress $\sigma_{\theta}$, along the outer perimeter of the CSR (i.e., along the locus E'AE, marked green in the sketch embedded in Fig. 13) is plotted for four different values of the $\varrho$-ratio. It is observed that for high $\varrho$-values the specific stress component is positive (i.e., of tensile nature) almost all along the CSR's outer perimeter. For example, for $\varrho=10.0$ no compressive stress appears for the whole range of $\theta$-values $\left(-90^{\circ}<\theta<90^{\circ}\right)$. Gradually, with decreasing $\varrho$, stresses of compressive nature appear also. The angle $\theta$, at which the transverse stress is zeroed, strongly depends on $\varrho$.

It is, also, worth noticing from Fig. 13 that, for high $\varrho$-values, the distribution of $\sigma_{\theta}$, is characteristically flat almost all along the outer perimeter of the CSR. It is mentioned, for example, that for $\varrho=10.0$ the value of $\sigma_{\theta}$ varies in a relatively narrow 
interval from about $37 \mathrm{MPa}$ at $\theta=0^{\circ}$ to about $30 \mathrm{MPa}$ for $\theta=60^{\circ}$. This flatness is lost with decreasing $\varrho$-values and the distribution of the transverse normal stress tends to obtain a bell-shaped form.

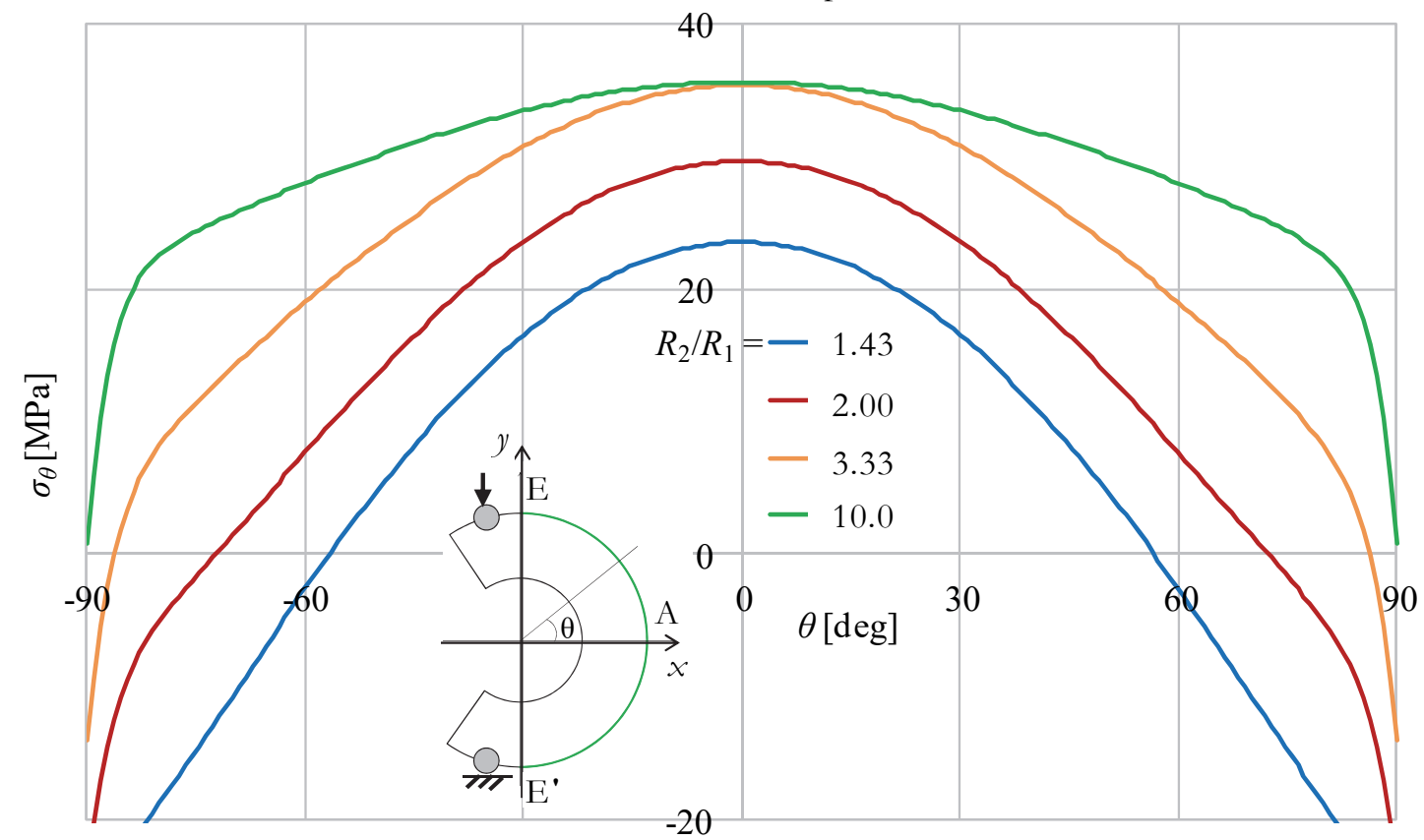

Figure 13: The polar distribution of the transverse normal stress along the outer perimeter of the CSR for various $\varrho$-values.

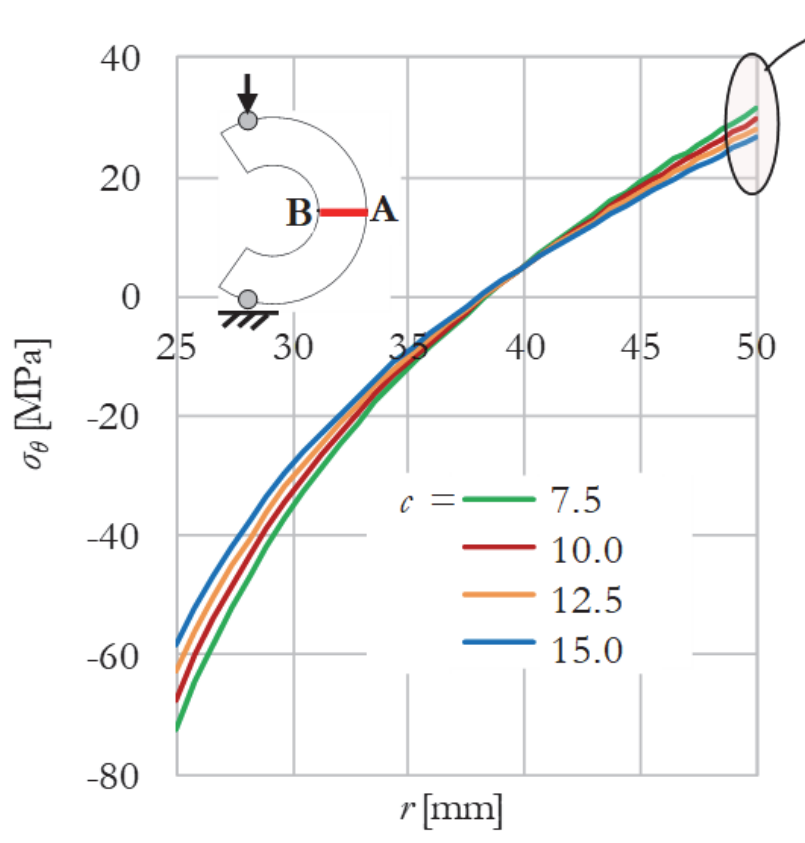

(a)

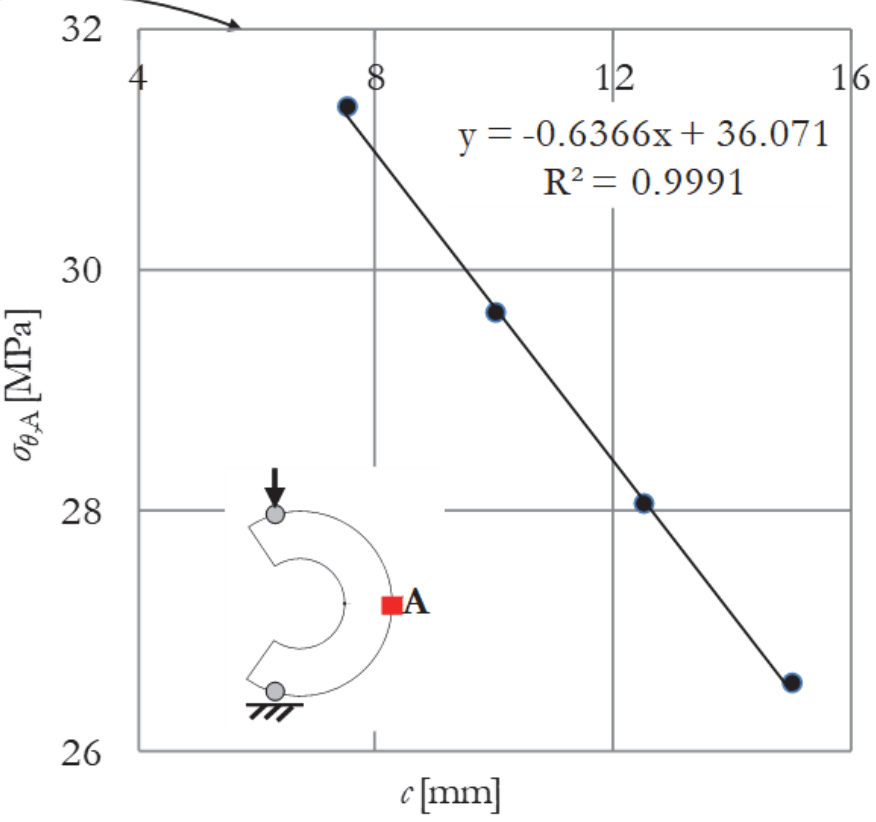

(b)

Figure 14: (a) The variation of the transverse stress along locus $A B$ for four different values of the eccentricity $c$ (in $\mathrm{mm}$ ); (b) The dependence of the maximum tensile stress (i.e., the stress developed at point A) on eccentricity $c$.

The next parameter studied is the eccentricity, $c$, of the pair of compressive forces loading the CSR-specimen. To explore its role, the variation of the transverse stress, $\sigma_{\theta}$, along the critical locus $\mathrm{AB}$ is plotted in Fig. 14a, for four different values of $c$ within the $0.015 R_{2}<c<0.030 \mathrm{R}_{2}$ range (or, alternatively, in the $7.5 \mathrm{~mm}<c<15.0 \mathrm{~mm}$ range). It can be observed from this figure that, with increasing $c$-values, the magnitude of the maximum tensile stress developed (i.e., that at point $\mathrm{A}$ ) decreases (recall that the loading scheme consists of a constant vertical displacement imposed on the upper cylindrical rod) and the same is true for the magnitude of the maximum compressive stress (i.e., the one developed at point B). In other words, 
the slope of the plot is reduced with increasing $c$. Moreover, it is to be highlighted that all plots share a common point at about $r=0.8 \mathrm{R}_{2}$ for all values of $c$. On the other hand, the point at which the transverse stress, $\sigma_{\theta}$, is zeroed, appears depending (though very slightly) on the value of the parameter $c$.

The influence of $c$ on the magnitude of the maximum tensile stress developed (i.e., the quantity of utmost practical importance) is more vividly shown in Fig. 14b: At least for the range of $c$-values studied here, the relation between $\sigma_{\theta, \text { max }} \equiv \sigma_{\theta, \mathrm{A}}$ and $c$ is perfectly linear. From a quantitative point, increasing the value of $c$ by a factor of 2 , i.e., by $100 \%$ (from $7.5 \mathrm{~mm}$ to $15.0 \mathrm{~mm}$ ), results to a decrease of $\sigma_{\theta, \max }$ from about $31.3 \mathrm{MPa}$ to about $26.5 \mathrm{MPa}$, or in other words only by about $15 \%$, indicating the rather minor influence of $c$ on the quantity of importance (i.e., on $\sigma_{\theta, \mathrm{A}}$ ).

In Fig. 15 the polar distribution of the transverse stress $\sigma_{\theta}$, along the outer perimeter of the CSR (i.e., along the locus E'AE, marked green in the sketch embedded in Fig.15) is plotted for four different values of the eccentricity (the same with the ones considered in previous paragraph). All four curves are quite similar to each other with minor differences, of only quantitative nature. These differences are more or less negligible for the major portion of the range of $\theta$ considered and only for $\theta$-values approaching $\pm 90^{\circ}$ do these differences increase significantly. All plots share two common points, symmetric with respect to the $\theta=0^{\circ}$ line, located at about $\theta= \pm 48^{\circ}$.

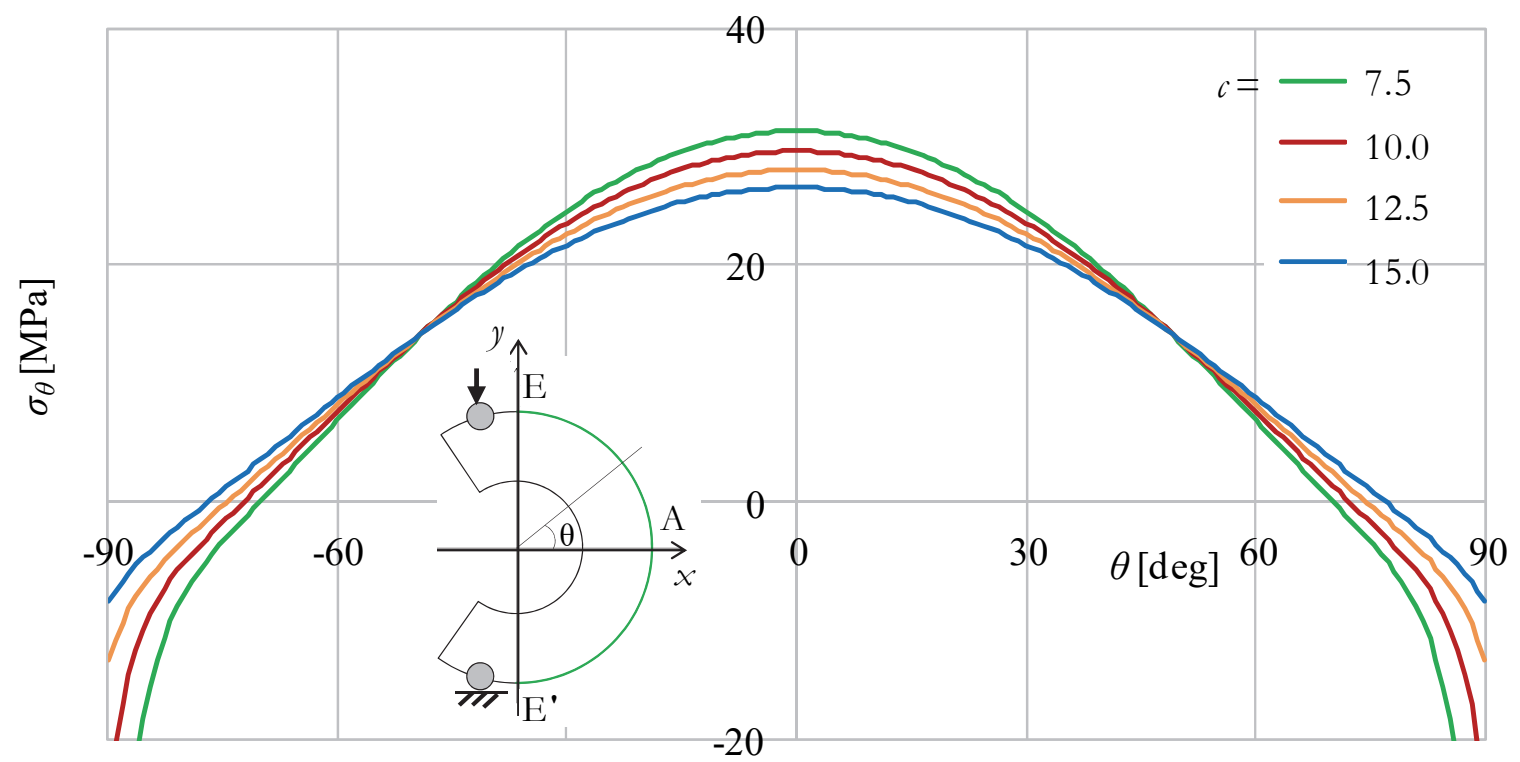

Figure 15: The polar distribution of the transverse normal stress along the outer perimeter of the CSR for various $c$-values (in mm).

\section{DISCUSSION AND CONCLUDING REMARKS}

A $\mathrm{n}$ alternative configuration for the laboratory determination of the tensile strength of brittle materials was proposed, consisting of a Circular Semi-Ring (CSR) subjected to diametral compression, either centrally or slightly eccentrically. The configuration is proposed as a possible substitute of the familiar standardized Brazilian-disc test (BDT), taking into account severe criticism against it, concerning the validity of its results and the relation of its outcome with the actual tensile strength as it is obtained from a direct tension test [3-5].

An analytic solution was introduced for the stress field developed in the CSR, based on the solution for the cut circular ring, following the approach introduced by Golovin [21] and Muskhelishvili [20]. The solution was checked against experimental data and the agreement was found very satisfactory. A three dimensional numerical model was then designed that permitted parametric exploration of the role of some geometrical and loading parameters on the distribution of stresses and their magnitude along various critical paths and strategic points of the CSR-specimens.

The data of the numerical model are here considered in juxtaposition to the respective ones of the analytic solution. This is achieved by plotting the transverse normal stress $\sigma_{\theta}$ along the critical path $\mathrm{AB}$, according to both approaches (numerical and analytical). The plots are implemented for the reference model $\left(R_{1}=25 \mathrm{~mm}, R_{2}=50 \mathrm{~mm}, 2 \mathrm{~h}=10 \mathrm{~mm}\right)$ and the results are exhibited in next Fig. 16. It is clear that the two approaches are extremely close to each other all along the locus considered. The maximum discrepancy is observed at $r=50 \mathrm{~mm}$ (i.e., at point A) and it does not exceed $7 \%$. 
The main advantage of the CSR-configuration (compared to the BDT one) is that the stress field, at the point of expected fracture (namely point A in Fig. 1a), includes a single component of tensile nature. Therefore, by determining the force, $P_{f}$, required to cause fracture of a CSR-specimen of given geometry, one can determine the respective stress at point A, which can be assumed as representing the tensile strength of the specimen's material.

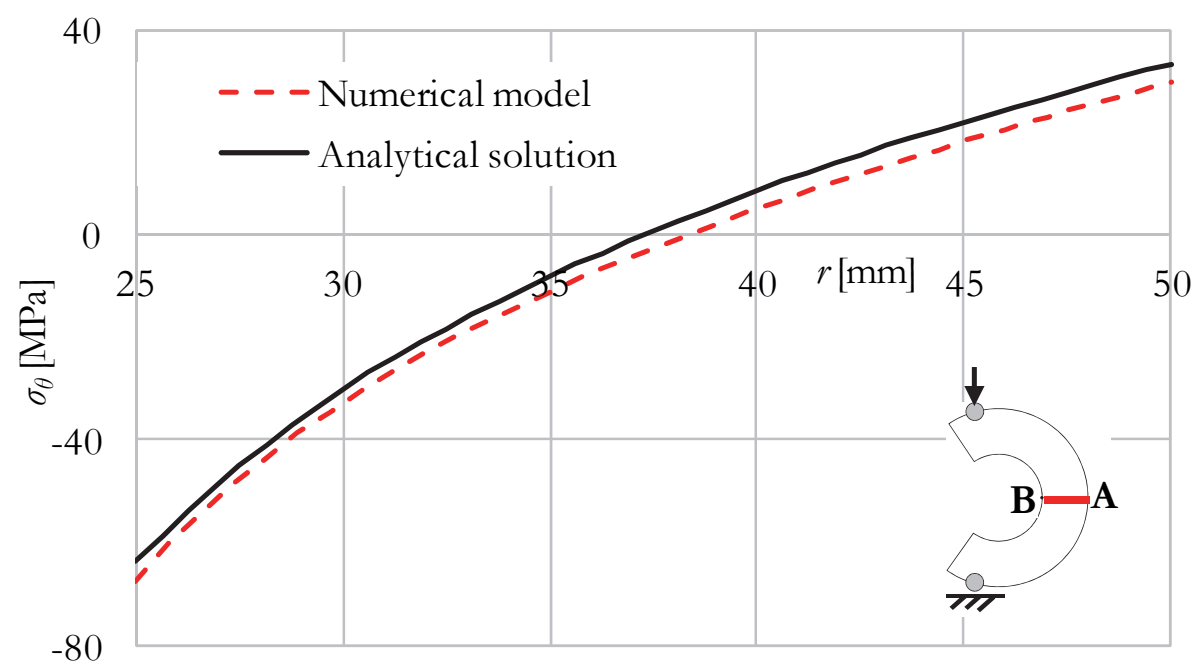

Figure 16: The distribution of the transverse normal stress along the critical locus $\mathrm{AB}$, according to the analytical solution and the data provided by the numerical model (for the reference configuration).

According to the results of the theoretical analysis described in previous sections, this critical stress is given as:

$$
\sigma_{\text {tensile }}^{\text {ultimate }}=\sigma_{\theta}^{\mathrm{A}}=\frac{P_{f}}{h}\left\{\frac{2 c\left(R_{2}^{2}-R_{1}^{2}-2 R_{1}^{2} \log \frac{R_{2}}{R_{1}}\right)}{\left(R_{1}^{2}-R_{2}^{2}\right)^{2}-4 R_{1}^{2} R_{2}^{2}\left(\log \frac{R_{2}}{R_{1}}\right)^{2}}+\frac{R_{2}^{2}-R_{1}^{2}}{R_{2}\left[R_{1}^{2}-R_{2}^{2}+\left(R_{1}^{2}+R_{2}^{2}\right) \log \frac{R_{2}}{R_{1}}\right]}\right\}
$$

Considering a CSR with $\varrho=2$ and $c=0.2 R_{2}$ the above equation is rewritten in terms of the outer radius, $R_{2}$, of the CSR as:

$$
\sigma_{\text {tensile }}^{\text {ultimate }}=\sigma_{\theta}^{\mathrm{A}}=k_{C S R} \frac{P_{f}}{R_{2}(2 h)}
$$

where $k_{C S R}$ is a numerical constant, depending on the pair of parameters $\varrho$ and $c$. For the above considered numerical values (i.e., $\varrho=2$ and $c=0.2 R_{2}$, it holds that $k_{C S R}=16.812$ ).

At this point, it is worth highlighting that, Eqn. (23) closely resembles the formula proposed by Mellor and Hawkes [14] for the tensile stress developed at the intersection of the loading diameter of a circular ring under diametral compression with the ring's inner boundary (which when the force applied causes fracture is, also, supposed to provide the tensile strength of the ring's material). This formula reads as:

$$
\left(\sigma_{\text {tensile }}^{\text {ultimate }}\right)_{\text {Circular ring }}=K \frac{P_{f}}{\pi \mathrm{R} t}=k_{\text {Circular ring }} \frac{P_{f}}{\mathrm{R} t}
$$

where, according to Mellor and Hawkes, $K$ is a numerical constant (depending on the ratio of the ring's radii), $t$ is the ring's thickness (it is recalled that, according to the notation of the present study, $t=2 h$ ) and $R$ its outer radius.

The above statement, i.e., that the CSR-test can successfully provide the tensile strength of the specimen's material, was experimentally assessed by submitting a number of CSR-specimens $\left(R_{1}=25 \mathrm{~mm}, R_{2}=50 \mathrm{~mm}, 2 h=10 \mathrm{~mm}\right)$, made of PMMA, to diametral compression. The average fracture force, $P_{f}$, of all successful tests of that protocol was equal to 
about $P_{f}=1.79 \mathrm{kN}$. Substituting this value of $P_{f}$ into Eqn. (22), the maximum tensile stress at point A (in other words the tensile strength of the CSR's material) was calculated equal to about $60.2 \mathrm{MPa}$. A preliminary protocol of direct tension tests with standardized dog-bone specimens (of the same batch from which the CSR-specimens were cut), provided a value for the tensile strength of the material equal to about $58.8 \mathrm{MPa}$, i.e., only $2.4 \%$ lower than that determined with the aid of the CSR-test. On the other hand, a series of tests with compact discs (again made of the same batch of material and having diameter $2 \mathrm{R}$ equal to $100 \mathrm{~mm}$ and thickness t equal to $10 \mathrm{~mm}$ ) subjected to Brazilian disc test [1, 2], in accordance with the ISRM suggestion, provided a fracture force equal to $P_{B r}=87.65 \mathrm{kN}$. Adopting Hondros' familiar formula [3], the respective tensile strength is calculated equal to $\sigma_{B r}=P /(\pi R t)=55.8 \mathrm{MPa}$, i.e., $5.1 \%$ lower than the value provided by the direct tension tests.

The above mentioned inconsistency, between the data of the direct tension test and the CSR-test, even though it is relatively small, could be justified by taking into account the role of another parameter (which was ignored up to this point), namely the thickness of the CSR-specimens. In fact, the specimens tested in the experimental protocol had a thickness of $10 \mathrm{~mm}$. They were neither very thin (plane stress) nor very thick (plane strain) for obvious practical reasons. The role of the specific parameter cannot be analytically explored (the analysis described is not actually three dimensional and the configurations considered must be characterized either as plane stress or plane strain). Inevitably testing specimens of moderate thickness introduces some discrepancies.

In this context, the role of thickness is here explored by taking advantage of the numerical model (previously validated), and more specifically of the respective reference configuration. In Fig. 17a the variation of the horizontal displacement $u_{x} \equiv u$ along the axis of symmetry of the CSR-specimen (locus AB) is plotted for both the front face (identical to the rear one) and the central section of the three dimensional numerical model. The difference between the two plots are by no means negligible, however, it is almost constant all along the locus considered. In the same figure the results of the analytical solution for the variation of $u_{x} \equiv u$ along locus $\mathrm{AB}$ are plotted, assuming either 2D-plane stress or 2D-plane strain conditions. The difference between the two cases is, now, not constant along the AB locus. It is zeroed for $r=25 \mathrm{~mm}$ (inner contour of the CSR) and increases gradually until $r \approx 35 \mathrm{~mm}$. Then it starts decreasing until $r=50 \mathrm{~mm}$ (outer contour of the CSR).

The respective plots for the transverse stress developed along the AB locus are exhibited in Fig. 17b. As it is, perhaps, expected, the difference for the stresses between the central section and the two surface faces of the three dimensional numerical model are negligible. On the contrary, the difference between the plane strain and the plane stress assumptions is quite considerable, approaching $13 \%$ at points $\mathrm{A}$ and $\mathrm{B}$ (the points at which the maximum difference is observed). More specifically, the maximum tensile stress (i.e., that at point A) is equal to about $29.7 \mathrm{MPa}$ for the $2 \mathrm{D}$-plane stress scheme, while for the respective $2 \mathrm{D}$-plane strain scheme it is equal to about $33.6 \mathrm{MPa}$.

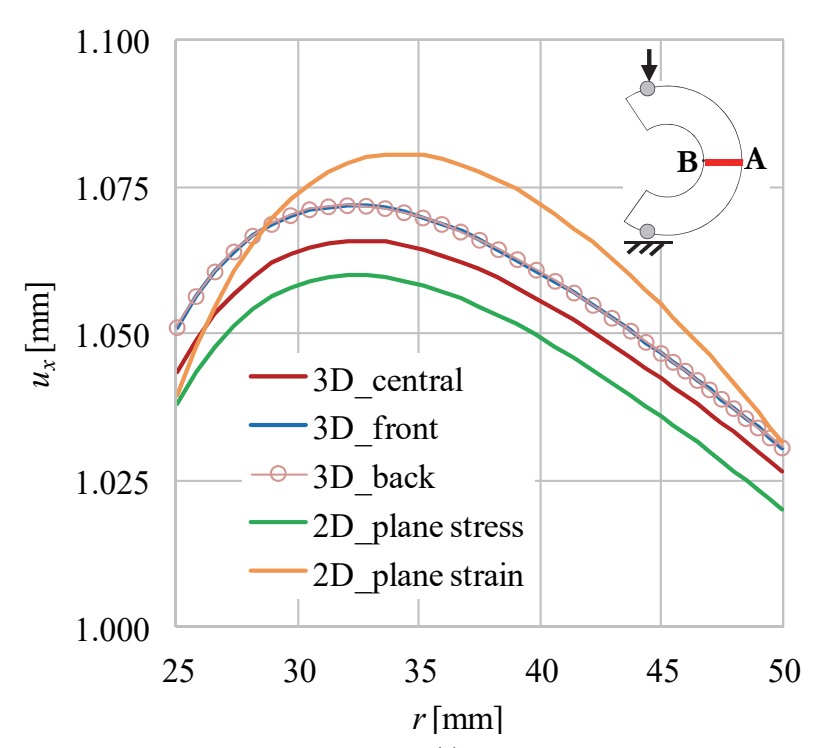

(a)

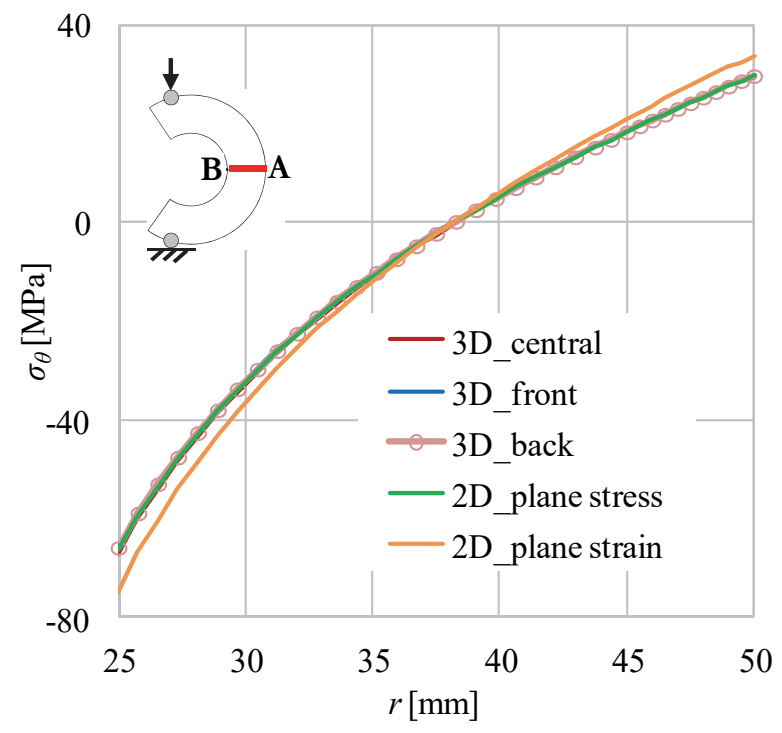

(b)

Figure 17: The variation of (a) the horizontal displacement $u_{x} \equiv u$ and (b) the normal transverse stress along the AB locus, according to the reference configuration of the numerical model, assuming either three dimensional conditions (the plots are realized for both the front and rear surfaces and also the central section) or two dimensional conditions (plane stress and plane strain). 
The above analysis clearly indicates that the thickness of the CSR-specimens plays an important role, which should not be ignored in case the CSR-test is to be used for the determination of the tensile strength. Given that the analytic solution cannot be applied for intermediate states between plane-stress and plane-strain conditions, Eqs.(22, 23) must be handled with caution. Research in progress [27] aims to provide correction factors, following a hybrid analytic-numerical approach.

Coming to an end, it can be safely stated that the CSR-test is indeed a flexible alternative for the determination of the tensile strength of brittle materials, the potentialities of which should be further explored, both experimentally (for specimens made of rocks and rock-like materials) and numerically (in the direction of quantifying the role of additional parameters).

\section{REFERENCES}

[1] Carneiro, F. L. L. B. (1943). A new method to determine the tensile strength of concrete, Proc. $5^{\text {th }}$ Meeting of the Brazilian Association for Technical Rules, 3d. Section, 16 September 1943, pp. 126-129 (in Portuguese).

[2] Akazawa, T. (1943). New test method for evaluating internal stress due to compression of concrete (the splitting tension test) (part 1), J. Japan Soc. Civil Engrs, 29, pp. 777-787.

[3] Hondros, G. (1959). The evaluation of Poisson's ratio and the modulus of materials of a low tensile resistance by the Brazilian (indirect tensile) test with particular reference to concrete, Aust. J. Appl. Sci., 10, pp. $243-268$.

[4] Markides, Ch. F., Pazis, D.N., Kourkoulis, S. K. (2010). Closed full-field solutions for stresses and displacements in the brazilian disk under distributed radial load, Int. J. Rock Mech. Min. Sci, 47(2), pp. 227-237.

[5] Markides, Ch. F., Kourkoulis, S. K. (2012). The stress field in a standardized Brazilian disc: The influence of the loading type acting on the actual contact length, Rock Mech. Rock Eng, 45(2), pp. 145-158.

[6] Kourkoulis S. K., Markides, Ch. F., Chatzistergos, P. E. (2012). The Brazilian disc under parabolically varying load: Theoretical and experimental study of the displacement field, Int. J. Solids Struct., 49(7-8), pp. 959-972.

[7] Markides, Ch. F., Pazis, D.N., Kourkoulis, S. K. (2010). The Brazilian disc under non uniform distribution of radial pressure and friction, Int. J. Rock Mech. Min. Sci, 50, pp. 47-55.

[8] Kourkoulis, S. K., Markides, Ch. F., Hemsley, J. A. (2013). Frictional stresses at the disc-jaw interface during the standardized execution of the Brazilian disc test, Acta Mechanica, 224(2), pp. 255-268.

[9] Kourkoulis, S. K., Markides, Ch. F., Chatzistergos, P. E. (2012). The standardized Brazilian disc test as a contact problem, Int. J. Rock Mech. Min. Sci, 57, pp.132-141.

[10] Hobbs, D. W. (1964). The tensile strength of rocks, Int. J. Rock. Mech. Min. Sci., 1, pp. 385-396.

[11] Fairhurst, C. (1964). On the validity of the 'Brazilian' test for brittle materials, Int. J. Rock Mech. Min. Sci., 1, pp. 535-546.

[12] Hobbs, D. W. (1965). An assessment of a technique for determining the tensile strength of rock, Brit. J. Appl. Phys., 16, pp. 259-268.

[13] Hooper, J. A. (1971). The failure of glass cylinders in diametral compression. J. Mech. Phys. Solids, 19, pp. 179-200.

[14] Mellor, M., Hawkes, I. (1971). Measurement of tensile strength by diametral compression of discs and annuli, Eng. Geol., 5, pp. 173-225.

[15] Ripperger, E., Davis, N. (1947). Critical stresses in a circular ring (Paper no 2308), Trans. Am. Soc. Civil Engrs, 112, pp. 619-627.

[16] Jaeger, J.C., Hoskins, E. R. (1966). Stresses and failure in rings of rock loaded in diametral tension or compression. Brit. J. Appl. Phys., 17, pp. 685-692.

[17] Wang, Q. Z., Jia, X. M., Kou, S. Q., Zhang, Z. X., Lindqvist, P. A. (2004). The flattened Brazilian disc specimen used for testing elastic modulus, tensile strength and fracture toughness of brittle rocks: analytical and numerical results. Int. J. Rock Mech. Min. Sci., 41(2), pp. 245-253.

[18] Kuruppu, M. D., Obara, Y., Ayatollahi, M. R., Chong, K. P., Funatsu, T. (2014). ISRM-suggested method for determining the mode I static fracture toughness using semi-circular bend specimen. Rock Mech Rock Eng, 47(1), pp. 267-274.

[19] ASTM E399 - 90 (1997). Standard Test Method for Plane-Strain Fracture Toughness of Metallic Materials - A5. Special Requirements for the Testing of the Arc-Shaped Tension Specimen.

[20] Muskhelishvili, N. I. (1963). Some Basic Problems of The Mathematical Theory of Elasticity, Noordhoff, Groningen.

[21] Golovin, Kh. (1882). A static problem of the elastic body. Minutes of the Technological Institute, St. Petersburg, 1880-1881, St. Petersburg.

[22] Love, A. E. H. (1927). A Treatise on the Mathematical Theory of Elasticity, $4^{\text {th }}$ ed., Cambridge, 1927. 
[23] Timpe, A. (1905). Probleme der Spannungsverteilung in ebenen Systemen, einfach gelöst mit Hilf der Airyschen Function, Zeitschrift für Mathematik und Physik, 52, pp. 348-383.

[24] Volterra, V. (1907). Sur l' équilibre des corps élastiques multiplement connexes. Annales scientifiques de l' Éqole Normale Supérieure, 24, pp. 401-517.

[25] Markides, Ch. F., Pasiou, E. D., Kourkoulis, S. K. (2018), A preliminary study on the potentialities of the Circular Semi-Ring test, by Procedia Structural Integrity, 9, pp. 108-115.

[26] Pazis, D. N., Agioutantis, Z., Kourkoulis S. K. (2011). The optical method of reflected caustics applied for a plate with a central hole: Critical points and limitations, Strain, 47(6), pp. 489-498.

[27] Kourkoulis, S. K., Pasiou, E. D., Markides, Ch. F. (2018). The role of geometrical parameters on the results of the circular semi-ring test (to be submitted). 